\title{
Poisson Disorder Problem with Exponential Penalty for Delay
}

\author{
Erhan Bayraktar \\ Department of Mathematics, University of Michigan, Ann Arbor, Michigan 48109, \\ erhan@umich.edu, http://www-personal.umich.edu/erhan/ \\ Savas Dayanik \\ Department of Operations Research and Financial Engineering, Princeton University, Princeton, New Jersey 08544, \\ sdayanik@princeton.edu, http://www.princeton.edu/ sdayanik/
}

\begin{abstract}
We solve the Poisson disorder problem when the delay is penalized exponentially. Our objective is to detect as quickly as possible the unobservable time of the change (or disorder) in the intensity of a Poisson process. The disorder time delimits two different regimes in which one employs distinct strategies (e.g., investment, advertising, manufacturing). We seek a stopping rule that minimizes the frequency of false alarms and an exponential (unlike previous formulations, which use a linear) cost function of the detection delay. In the financial applications, the exponential penalty is a more apt measure for the delay cost because of the compounding of the investment growth. The Poisson disorder problem with a linear delay cost was studied by Peskir and Shiryaev [2002. Solving the Poisson Disorder Problem. Advances in Finance and Stochastics. Springer, Berlin, Germany, 295-312], which is a limiting case of ours.
\end{abstract}

Key words: Poisson disorder problem; quickest detection; optimal stopping; differential-delay equations MSC2000 subject classification: Primary: 62L10; secondary: 62L15, 62C10, 60G40

OR/MS subject classification: Primary: statistics: Bayesian; estimation; secondary: dynamic programming/optimal control: applications

History: Received March 25, 2005; revised August 29, 2005, and January 30, 2006.

1. Introduction. In this paper, we address a change-detection problem involving Poisson processes. Suppose that we observe a Poisson process $X=\left\{X_{t} ; t \geq 0\right\}$ whose intensity changes from $\lambda_{0}$ to $\lambda_{1}$ at some random time $\theta$. The "disorder time" $\theta$ is unobservable, but has a known a priori probability distribution: it equals zero with probability $\pi \in[0,1)$ and has exponential distribution with rate $\lambda$ given that it is positive. The parameters $\lambda, \lambda_{0}, \lambda_{1}$ are known positive constants. The Poisson disorder problem is to detect the change-time $\theta$ by using the observations of $X$. Here, we are interested in the best detection rule which minimizes the expected sum of the frequency of the false alarms and an exponential cost function of the detection delay.

The change-detection and sequential hypothesis testing problems about the drift of a Wiener process have been extensively studied; see, e.g., Shiryaev [17, 15, Chapter IV], Beibel [3, 2], Karatzas [8], Moustakides [10]. Similar questions for the intensity of a Poisson process also draw significant attention, because Poisson processes are often used to model abrupt changes, such as sudden price movements in stock markets, changes in credit ratings due to defaults, changes in the intensity of earthquakes, product failures in a manufacturing system, etc.

Particularly, the Poisson disorder problem with linear penalty functions of the delay has been investigated well; see, e.g., Galchuk and Rozovsky [7], Davis [6], Peskir and Shiryaev [12, 11]. However, in many applications the cost of the lost opportunity due to the detection delay exponentiates with the delay time. Therefore, it is captured better by an exponential penalty function than by a linear one.

As a simple motivating example from quality control, let us consider an assembly line whose finished products are continuously inspected for defects. A sudden upward shift (e.g., from low $\lambda_{0}$ to high $\lambda_{1}$ ) in the rate of the number of defective items (the observation process $X$ ) may have an assignable cause and warrant an investigation at some fixed cost. A good control policy should balance the costs of false alarm (due to unnecessary inspection) and detection delay (due to lost production time and raw materials, scrapping or recycling, etc.).

A firm often measures its financial losses and gains by compounding those at its own internal rate of return (IRR). Let us denote our firm's IRR by $\alpha$. Typically the production rate of an assembly line is constant, say one item per unit time. Suppose also that a defective item costs one dollar. If the production system goes out of control at time $\theta$, and an alarm is given at some later time $\tau$, then every $d t$ units of defective items at each $t \in[\theta, \tau)$ will cost $\exp \{\alpha(\tau-t)\} d t$ at the detection time $\tau$. Therefore, total cost of detection delay equals

$$
1_{\{\tau>\theta\}} \int_{\theta}^{\tau} e^{\alpha(\tau-t)} d t=1_{\{\tau>\theta\}} \int_{0}^{\tau-\theta} e^{\alpha t} d t=\int_{0}^{(\tau-\theta)^{+}} e^{\alpha t} d t=\frac{1}{\alpha}\left[e^{\alpha(\tau-\theta)^{+}}-1\right],
$$

and is an exponential function of the detection delay time $(\tau-\theta)^{+}$. In addition, if each false alarm costs $1 /(c \alpha)$ dollars on average, then an optimal alarm time $\tau$ should minimize the expected total inspection cost, which is proportional to

$$
\mathbb{P}\{\tau<\theta\}+c \mathbb{E}\left[e^{\alpha(\tau-\theta)^{+}}-1\right],
$$


i.e., the alarm time $\tau$ should solve optimally some Poisson disorder problem with an exponential penalty for delay.

The only alternative to exponential delay penalty in the literature (see, e.g., Galchuk and Rozovsky [7], Davis [6], Peskir and Shiryaev [12]) is the linear delay penalty, as in

$$
\mathbb{P}\{\tau<\theta\}+c \mathbb{E}\left[(\tau-\theta)^{+}\right] .
$$

From decision theoretic point of view, the penalty $\mathbb{E}\left[(\tau-\theta)^{+}\right]$is the choice of a risk-neutral decision maker. Indeed, if the risks implied by the fluctuations in the delay time $(\tau-\theta)^{+}$are important to a decision maker, then the linear penalty $\mathbb{E}\left[(\tau-\theta)^{+}\right]$falls short.

On the other hand, the exponential penalty $\mathbb{E}\left[e^{\alpha(\tau-\theta)^{+}}-1\right]$ not only captures the variability of the delay time, but also reflects the risk-sensitivity of a risk-averse decision maker; see Whittle [18, 19]. By adjusting the parameter $\alpha$, the decision maker can tune the exponential penalty to his/her risk preferences. To see these, let us replace in (2) the linear penalty $\mathbb{E}\left[(\tau-\theta)^{+}\right]$with the exponential penalty $(1 / \alpha) \mathbb{E}\left[e^{\alpha(\tau-\theta)^{+}}-1\right]$ and use the identity (see, for example, Bensoussan [5, p. 54])

$$
e^{\alpha x}=1+\alpha x+\alpha^{2} x^{2} \int_{0}^{1} \int_{0}^{s} e^{r \alpha x} d r d s, \quad x \geq 0
$$

to rewrite it. We obtain

$$
\mathbb{P}\{\tau<\theta\}+\frac{c}{\alpha} \mathbb{E}\left[e^{\alpha(\tau-\theta)^{+}}-1\right]=\mathbb{P}\{\tau<\theta\}+c \mathbb{E}\left[(\tau-\theta)^{+}\right]+c \alpha \mathbb{E}\left[\left((\tau-\theta)^{+}\right)^{2} \int_{0}^{1} \int_{0}^{s} e^{r \alpha(\tau-\theta)^{+}} d r d s\right] .
$$

Hence, the exponential penalty on the left accounts for the losses in (2) as well as the effect of the second-order terms in the delay time. For large values of $\alpha$, every alarm time $\tau$ causing high variations in the delay time $(\tau-\theta)^{+}$is now punished severely according to (3). For small values of $\alpha$, the punishment is lesser, and we retrieve the risk-neutral (linear) case (2) if we let $\alpha$ go to zero. Hence, the exponential penalty contains the linear penalty as a sub case and allows the risk preferences to be added to the analysis by a natural mechanism.

The importance of exponential delay penalty was recognized first by Poor [13], who solved a quickestdetection problem with exponential delay cost in the discrete-time setting. Later, Beibel [3] solved the Wiener disorder problem with the same cost function. The Poisson disorder problem with an exponential penalty function of the detection delay is studied for the first time in this paper, to the best of our knowledge.

To solve the Poisson disorder problem, we first show that it is equivalent to an optimal stopping problem for a two-dimensional jump process $(\Pi, \Phi)$ in $[0,1] \times \mathbb{R}_{+}$. For every $t \geq 0, \Pi_{t}$ is the conditional probability that the change occurred at or before time $t$ given the past observations of $X$. On the other hand, $\Phi_{t}$ is essentially the likelihood-ratio process $\Psi_{t} \triangleq \Pi_{t} /\left(1-\Pi_{t}\right)$ with an adjustment, adapted to the history of $X$, reflecting the exponential detection delay cost; see (7). The optimal stopping problem is reduced to a free-boundary problem involving a differential delay equation. By means of a key verification lemma, one solution of the free-boundary problem is identified as the value function of the optimal stopping problem. The optimal stopping rule turns out a threshold type for the process $\Phi$ regardless of $\Pi$ : declare that the disorder happened at or before time $t \geq 0$ as soon as $\Phi_{t}$ exceeds a suitable threshold (constant over time).

We characterize the optimal threshold and the value function of the optimal stopping problem. To calculate the threshold and the value function, we describe an efficient numerical method using bisection search on the real line and finite-difference method for differential-delay equations.

Our systematic numerical method also complements the work of Peskir and Shiryaev [12], whose problem is a limiting case of ours. Let us also mention that Beibel [3] reduced the Wiener disorder problem with exponential penalty function to a similar optimal stopping problem and solved it as a generalized parking problem. Beibel's approach relies on the continuity of the paths of the process $\Pi$. For the Poisson disorder problem, the process $\Pi$ has jumps, and the related optimal stopping problem cannot be formulated as a generalized parking problem.

In the next section, we give a precise description of our problem and formulate the equivalent optimal stopping problem in (6)-(8). The latter is solved, and an optimal Bayes rule and the minimum Bayes cost function are determined in $\S 3$; see Propositions 3.1-3.4. To calculate the optimal decision rules, numerical methods are also described; they are illustrated on examples in Figures 2 and 3 in $\$ 3$. Long proofs are presented in $\S 4$ and in the appendix.

Let us remark that our analysis of the free-boundary problem might be useful to solve other quickest-detection problems; see, for example, Bayraktar et al. [1] for an application to "standard" Poisson disorder problems. 
2. The problem. Let $\left(\Omega, \mathscr{F}, \mathbb{P}_{\pi}\right)$ be a probability space hosting two independent Poisson processes $X^{0}=$ $\left(X_{t}^{0}\right)_{t \geq 0}$ and $X^{1}=\left(X_{t}^{1}\right)_{t \geq 0}$ with rates $\lambda_{0}$ and $\lambda_{1}$, respectively, and a random variable $\theta$, independent of the processes $X^{0}$ and $X^{1}$, having the distribution

$$
\mathbb{P}_{\pi}\{\theta=0\}=\pi \quad \text { and } \quad \mathbb{P}_{\pi}\{\theta>t\}=(1-\pi) e^{-\lambda t}, \quad t \geq 0 .
$$

The processes $X^{0}, X^{1}$ and the random variable $\theta$ are unobservable. The observation process is

$$
X_{t}=\int_{0}^{t} 1_{\{s \leq \theta\}} d X_{s}^{0}+\int_{0}^{t} 1_{\{s>\theta\}} d X_{s}^{1}, \quad t \geq 0,
$$

with the natural filtration $\mathscr{F}^{X}=\left(\mathscr{F}_{t}^{X}\right)_{t \geq 0}$ (modified suitably to make it satisfy the usual conditions) and $\mathscr{F}_{\infty}^{X} \triangleq$ $\bigvee_{t \geq 0} \mathscr{F}_{t}^{X}$. For every $\mathscr{F}^{X}$-stopping time $\tau$ (sometimes, we write $\tau \in \mathscr{F}^{X}$ ), the associated Bayes error

$$
R_{\tau}(\pi) \triangleq \mathbb{P}_{\pi}\{\tau<\theta\}+c \mathbb{E}_{\pi}\left[e^{\alpha(\tau-\theta)^{+}}-1\right]
$$

is the sum of the probability of the false alarm $\mathbb{P}_{\pi}(\tau<\theta)$ and the expected exponential delay penalty $c \mathbb{E}_{\pi}\left[e^{\alpha(\tau-\theta)^{+}}-1\right]$ for some known positive constants $c$ and $\alpha$. The Poisson disorder problem is to find an $\mathscr{F}^{X}$-stopping time $\tau^{*}$ as close to the disorder time $\theta$ as possible in the sense that, if such a stopping time exists, it achieves the minimum Bayes error

$$
V(\pi) \triangleq \inf _{\tau} R_{\tau}(\pi), \quad \forall \pi \in[0,1]
$$

where the infimum is taken over all $\mathscr{F}^{X}$-stopping times $\tau$. In fact, it is enough to take the infimum in (6) only over the $\mathscr{F}^{X}$-stopping times having finite expectations (this will be useful later in establishing the relationship (17)). Indeed, if $\tau$ is an $\mathscr{F}^{X}$-stopping time and $\mathbb{E}_{\pi} \tau=\infty$, then the Jensen's inequality implies that $R_{\tau} \geq c \mathbb{E}_{\pi}\left[e^{\alpha(\tau-\theta)^{+}}-1\right] \geq c\left[\exp \left\{\alpha\left(\mathbb{E}_{\pi} \tau-\mathbb{E}_{\pi} \theta\right)^{+}\right\}-1\right]=\infty>1-\pi=R_{0}(\pi) \geq V(\pi)$; i.e., $\tau$ cannot attain the infimum in (6). In terms of the $\mathscr{F}^{X}$-adapted processes

$$
\Pi_{t} \triangleq \mathbb{P}_{\pi}\left\{\theta \leq t \mid \mathscr{F}_{t}^{X}\right\} \quad \text { and } \quad \Phi_{t} \triangleq \frac{\mathbb{E}_{\pi}\left[1_{\{\theta \leq t\}} e^{\alpha(t-\theta)^{+}} \mid \mathscr{F}_{t}^{X}\right]}{1-\Pi_{t}}, \quad t \geq 0
$$

the Bayes error $R_{\tau}(\pi)$ in (5) can be expressed as

$$
R_{\tau}(\pi)=1-\pi+\mathbb{E}_{\pi} \int_{0}^{\tau}\left(1-\Pi_{s}\right)\left(c \alpha \Phi_{s}-\lambda\right) d s
$$

for every $\mathscr{F}^{X}$-stopping time $\tau$ (see p. 231 for the proof). We interpret the process $\Phi_{t}$ in $(7)$ as the weighted likelihood-ratio process-with the exponential delay cost as the weight-because of its resemblance to the well-known likelihood-ratio process $\Psi_{t} \triangleq \Pi_{t} /\left(1-\Pi_{t}\right)$, the sufficient statistic in many statistical detection and hypothesis testing problems. In our problem, $\Phi$ also turns out the sufficient statistic in the sense that it completely determines the optimal detection rule. The standard applications of Bayes' theorem and the chain rule (see $\S$ A.1 in the appendix) reveal the dynamics of the jump processes $\Pi, \Psi$, and $\Phi$ as

$$
\begin{aligned}
& d \Pi_{t}=\left[\lambda-\left(\lambda_{1}-\lambda_{0}\right) \Pi_{t}\right]\left(1-\Pi_{t}\right) d t+\frac{\left(\lambda_{1}-\lambda_{0}\right) \Pi_{t-}\left(1-\Pi_{t-}\right)}{\lambda_{0}\left(1-\Pi_{t-}\right)+\lambda_{1} \Pi_{t-}} d X_{t}, \quad \Pi_{0}=\pi, \\
& d \Psi_{t}=\left[\lambda+\left(\lambda-\lambda_{1}+\lambda_{0}\right) \Psi_{t}\right] d t+\left(\lambda_{1} / \lambda_{0}-1\right) \Psi_{t-} d X_{t}, \quad \Psi_{0}=\pi /(1-\pi), \\
& d \Phi_{t}=\left[\lambda+\left(\lambda+\alpha-\lambda_{1}+\lambda_{0}\right) \Phi_{t}\right] d t+\left(\lambda_{1} / \lambda_{0}-1\right) \Phi_{t-} d X_{t}, \quad \Phi_{0}=\pi /(1-\pi) .
\end{aligned}
$$

Evidently, the processes $\Pi, \Psi$, and $\Phi$ are strongly Markovian. With the new look of the Bayes risk in (8), the quickest-detection problem of (6) is an optimal stopping problem for the two-dimensional Markovian jump process $(\Pi, \Phi)$. In the next section, we shall formulate the optimal stopping problem as a free-boundary problem involving the infinitesimal generator of $(\Pi, \Phi)$ and solve the latter.

3. Free boundary problem and its solution. We start this section with an observation. For every real number $\phi$, let us denote the exit time of $\Phi$ out of the interval $[0, \phi)$ by

$$
\tau_{\phi} \triangleq \inf \left\{t \geq 0: \Phi_{t} \geq \phi\right\} \quad(\inf \varnothing \equiv+\infty)
$$


and define for future reference

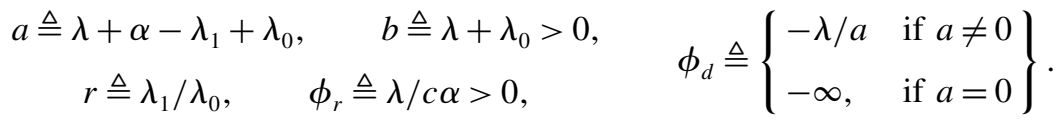

The drift $\phi \mapsto \lambda+a \phi$ of $\Phi$ in (11) changes its sign at $\phi=\phi_{d}$ ("d" for drift); the sign of the integrand in (8) is determined by the function $\phi \mapsto c \alpha \phi-\lambda$ whose sign changes at $\phi=\phi_{r}$ ("r" for reward). As clearly seen from (8), the Bayes risk $R_{\tau}(\pi)$ decreases as long as the process $\Phi$ stays in $\left[0, \phi_{r}\right)$. Therefore, it is not optimal to stop before $\Phi$ leaves $\left[0, \phi_{r}\right)$.

Lemma 3.1. If an $\mathscr{F}^{X}$-stopping time $\tau$ is optimal for $(6,8)$, then so is $\tau \vee \tau_{\phi_{r}}$, where $\phi_{r}$ is as in (13).

Proof. For every $\pi \in[0,1]$, (8) implies $R_{\tau \vee \tau_{\phi_{r}}}(\pi)=R_{\tau}(\pi)+\mathbb{E}_{\pi} \int_{\tau}^{\tau \vee \tau_{\phi_{r}}}\left(1-\Pi_{s}\right)\left(c \alpha \Phi_{s}-\lambda\right) d s \leq R_{\tau}(\pi)=$ $V(\pi)$, i.e., $R_{\tau \vee \tau_{\phi_{r}}}(\pi)=V(\pi)$.

Hence, we can restrict our search for an optimal stopping time to those $\mathscr{F}^{X}$-stopping times $\tau$ satisfying $\tau \geq \tau_{\phi_{r}}, \mathbb{P}_{\pi}$-a.s. for all $\pi \in[0,1]$. From this observation and the behavior of the paths of the process $\Phi$, our first result follows.

Proposition 3.1 (CASE I). Let $\lambda_{1} \geq \lambda_{0}$. If $\phi_{d}<0$ or $0<\phi_{d} \leq \phi_{r}$, then the stopping time $\tau_{\phi_{r}}$ is optimal for $(6,8)$, where $\phi_{r}$ and $\phi_{d}$ are as in (13).

Let $\sigma_{0} \equiv 0$ and $\sigma_{n} \triangleq \inf \left\{t>\sigma_{n-1}: \Phi_{t}-\Phi_{t-}>0\right\}$ be the $n$th jump time of $\Phi$ for every $n \in \mathbb{N}$ (by convention, $\inf \varnothing=+\infty$ ). From (11), it is easy to obtain that

$$
\begin{gathered}
\Phi_{t}=\left\{\begin{array}{cc}
\phi_{d}+\left[\Phi_{\sigma_{n-1}}-\phi_{d}\right] \exp \left\{-\left(\lambda / \phi_{d}\right)\left(t-\sigma_{n-1}\right)\right\}, & \phi_{d} \neq-\infty \\
\Phi_{\sigma_{n-1}}+\lambda t, & \phi_{d}=-\infty
\end{array}\right\}, \quad \sigma_{n-1} \leq t<\sigma_{n}, \\
\Phi_{0} \in \mathbb{R}_{+} \quad \text { and } \quad \Phi_{\sigma_{n}}=\left(\lambda_{1} / \lambda_{0}\right) \Phi_{\sigma_{n}-}, \quad n \in \mathbb{N} .
\end{gathered}
$$

If $\phi_{d}<0$, then the paths of the process $\Phi$ always increase between jumps. If $\phi_{d}>0$, then $\phi_{d}$ is the mean-level to which the process $\Phi$ reverts between jumps; the difference $\Phi_{t}-\phi_{d}$ in (14) never vanishes before a jump, and $\Phi_{\sigma_{n}} \neq \phi_{d}$ for all $n>0$ almost surely. If $\phi_{d}>0$, then the state $\phi_{d}$ is an "entrance boundary" for $\Phi$ : it can start at $\phi_{d}$-and stays there until the first jump time, but never comes back to $\phi_{d}$ after it leaves.

If $\lambda_{1}>\lambda_{0}$, then $\Phi$ has positive jumps; $\phi_{d}$ might be negative or positive; see Figure 1(a, b). If $\lambda_{1}=\lambda_{0}$, then the process $\Phi$ never jumps; since $\phi_{d}$ is always defined and negative, the paths of $\Phi$ increase. If $\lambda_{1}<\lambda_{0}$, then $\Phi$ has negative jumps; since $\phi_{d}$ is always defined and negative, the paths of $\Phi$ increase between jumps; see Figure 1(c).

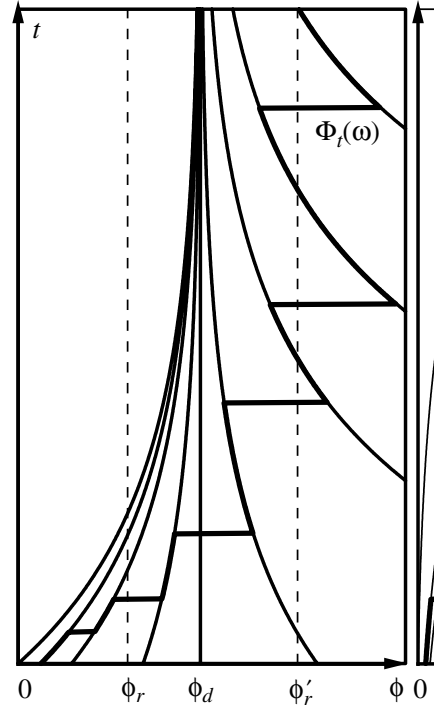

(a) $\lambda_{1}>\lambda_{0}, \phi_{d}>0$

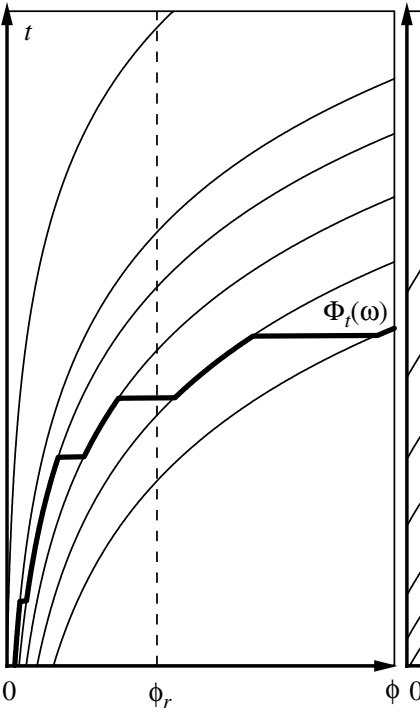

(b) $\lambda_{1}>\lambda_{0}, \phi_{d}<0$

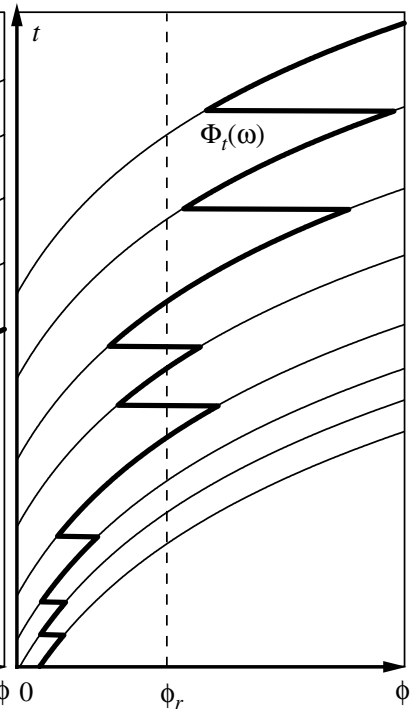

(c) $\lambda_{1}<\lambda_{0}\left(\phi_{d}<0\right)$

FIGURE 1. Sample paths of the process $\Phi$ of $(11,14)$. The process $\Phi$ has positive jumps if $\lambda_{1}>\lambda_{0}(\mathrm{a}, \mathrm{b})$, and negative jumps if $\lambda_{1}<\lambda_{0}$ (c). The paths increase between jumps if $\phi_{d}<0$ (b, c). Note from (13) that $\phi_{d}$ is always negative if $\lambda_{1}<\lambda_{0}$. If $\phi_{d}>0$, then $\Phi$ reverts to the mean-level $\phi_{d}$ between (positive) jumps (a). 
Proof of Proposition 3.1. In all three cases, the process $\Phi$ does not return to the interval $\left[0, \phi_{r}\right]$, once it leaves; see Figure 1(a, b). Therefore, $\tau_{\phi_{r}}$ is optimal.

In the remainder, we focus on $\lambda_{1}>\lambda_{0}, 0<\phi_{d}<\phi_{r}$ (Case II), and $\lambda_{1}<\lambda_{0}$ (Case III). In both cases, the process $\Phi$ returns to the interval $\left[0, \phi_{r}\right]$ with positive probability after every exit (see Figure $1(\mathrm{a}, \mathrm{c})$ ); the optimal stopping rule for $(6,8)$ turns out in the form of $\tau_{\phi}$ of (12) for some suitable $\phi>\phi_{r}$.

On $\left(\Omega, \mathscr{F}, \mathbb{P}_{\pi}\right)$, let the $\mathscr{F}^{X}$-adapted process $\tilde{\Phi}$ be the unique solution of the stochastic differential equation

$$
d \tilde{\Phi}_{t}=\left[\lambda+\left(\lambda+\alpha-\lambda_{1}+\lambda_{0}\right) \tilde{\Phi}_{t}\right] d t+\left(\lambda_{1} / \lambda_{0}-1\right) \tilde{\Phi}_{t-} d X_{t}, \quad \tilde{\Phi}_{0}=\phi \in[0,+\infty) .
$$

For every $(\pi, \phi) \in[0,1] \times \mathbb{R}_{+}$, we shall denote by $\mathbb{P}_{\pi, \phi}$ the probability measure $\mathbb{P}_{\pi}$ if $\mathbb{P}_{\pi}\left\{\tilde{\Phi}_{0}=\phi\right\}=1$. Let $\mathbb{E}_{\pi, \phi}$ be the expectation under $\mathbb{P}_{\pi, \phi}$, and introduce the auxiliary optimal stopping problem

$$
\tilde{V}(\pi, \phi) \triangleq \inf _{\tau} \mathbb{E}_{\pi, \phi} \int_{0}^{\tau}\left(1-\Pi_{s}\right)\left(c \alpha \tilde{\Phi}_{s}-\lambda\right) d s, \quad(\pi, \phi) \in[0,1] \times \mathbb{R}_{+},
$$

where the infimum is taken over all $\mathscr{F}^{X}$-stopping times having finite $\mathbb{P}_{\pi, \phi}$-expectations. Note from (11) and (15) that the finite-dimensional distribution of $(X, \Pi, \tilde{\Phi})$ under $\mathbb{P}_{\pi, \pi /(1-\pi)}$ is the same as that of $(X, \Pi, \Phi)$ under $\mathbb{P}_{\pi}$. Therefore, the value function of the original optimal stopping problem $(6,8)$ is given by

$$
V(\pi)=1-\pi+\tilde{V}(\pi, \pi /(1-\pi)), \quad \pi \in[0,1]
$$

ANSATZ. For a suitable function $g: \mathbb{R}_{+} \mapsto \mathbb{R}$ and a real number $\phi>\phi_{r}$, the value function $\tilde{V}$ and the optimal continuation region for (16) are in the forms of

$$
\tilde{V}(\pi, \phi)=(1-\pi) g(\phi), \quad(\pi, \phi) \in[0,1] \times \mathbb{R}_{+} \quad \text { and } \quad \mathbf{C}=[0,1] \times[0, \phi),
$$

respectively. The first exit time of $(\Pi, \tilde{\Phi})$ out of $\mathbf{C} \subset[0,1] \times \mathbb{R}_{+}$is optimal for (16).

It is obvious from (5), (6), and (8) that $0 \leq V(\pi) \leq 1-\pi$ for every $\pi \in[0,1]$. If the ansatz is true, then (17) and (18) imply that $0 \leq V(\pi)=1-\pi+(1-\pi) g(\pi /(1-\pi)) \leq 1-\pi$ for every $\pi \in[0,1]$; namely, $g(\cdot)$ is bounded, and

$$
-1 \leq g(\phi) \leq 0, \quad \forall \phi \in \mathbb{R}_{+} .
$$

The infinitesimal generator $\mathscr{A}$ of $(\Pi, \tilde{\Phi})$ coincides with the first order differential-difference operator in (67) acting on the functions in $\mathbf{C}^{1,1}\left([0,1] \times \mathbb{R}_{+}\right)$. If $g \in \mathbf{C}^{1}\left(\mathbb{R}_{+}\right)$, then the free-boundary problem associated with $(16,18)$ becomes

$$
\begin{gathered}
\mathscr{A}(1-\pi) g(y)=-(1-\pi)(c \alpha y-\lambda), \quad(\pi, y) \in[0,1] \times[0, \phi), \\
(1-\pi) g(y)=0, \quad(\pi, y) \in[0,1] \times[\phi, \infty)
\end{gathered}
$$

It is easily checked that $\mathscr{A}(1-\pi) g(y)=(1-\pi)\left[(\lambda+a y) g^{\prime}(y)-b g(y)+\lambda_{0} g(r y)\right]$ for every $(\pi, y) \in[0,1] \times \mathbb{R}_{+}$, where $a, b, r$ are defined as in (13). Therefore, $(20,21)$ simplifies to the one-dimensional free-boundary problem $\left(20^{\prime}, 21^{\prime}\right)$ below. The proof of the next lemma is given on page 232 after the supporting facts are established in $\S$ A.2 of the appendix.

Verification Lemma. Let $g: \mathbb{R}_{+} \mapsto(-\infty, 0]$ be a bounded, continuous and piecewise continuously differentiable function such that

$$
(\lambda+a y) g^{\prime}(y)-b g(y)+\lambda_{0} g(r y) \geq-c \alpha y+\lambda, \quad y \in \mathbb{R}_{+}
$$

whenever $g^{\prime}(y)$ exists. Then $\tilde{V}(\pi, y) \geq(1-\pi) g(y)$ for every $(\pi, y) \in[0,1] \times \mathbb{R}_{+}$. In addition, if $g \in \mathbf{C}\left(\mathbb{R}_{+}\right) \cap$ $\mathbf{C}^{1}\left(\mathbb{R}_{+} \backslash\left\{\phi_{d}, \phi\right\}\right)$ for some real number $\phi>\phi_{r}$ and

$$
\begin{gathered}
(\lambda+a y) g^{\prime}(y)-b g(y)+\lambda_{0} g(r y)=-c \alpha y+\lambda, \quad y \in\left(0, \phi_{d}\right) \cup\left(\phi_{d}, \phi\right), \\
g(y)=0, \quad y \in[\phi, \infty),
\end{gathered}
$$

then (18) holds, i.e., $\tilde{V}(\pi, y)=(1-\pi) g(y)$ for every $(\pi, y) \in[0,1] \times \mathbb{R}_{+} ;$the stopping time $\tau_{\phi} \triangleq \inf \{t \geq$ 0: $\left.\tilde{\Phi}_{t} \geq \phi\right\}$ has finite $\mathbb{P}_{\pi, \phi}$ expectation and is optimal for (16). 
Proposition 3.2 (CASEs II AND III). There exist unique real number $\phi^{*}>\phi_{r}$ and unique function $g: \mathbb{R}_{+} \mapsto$ $[-1,0]$ in $\mathbf{C}\left(\mathbb{R}_{+}\right) \cap \mathbf{C}^{1}\left(\mathbb{R}_{+} \backslash\left\{\phi_{d}, \phi^{*}\right\}\right)$ that satisfy (22), (20'), (21') with $\phi^{*}$ instead of $\phi$. The minimum Bayes error in (5) is

$$
V(\pi)=1-\pi+(1-\pi) g(\pi /(1-\pi)), \quad \forall \pi \in[0,1],
$$

and $\tau^{*} \triangleq \tau_{\phi^{*}}=\left\{t \geq 0: \Phi_{t} \geq \phi^{*}\right\}$ is a minimum Bayes stopping rule.

The next section is devoted to the proof of the existence and the uniqueness of $\phi^{*}$ and $g$. The rest of Proposition 3.2 follows from (17) and the verification lemma above. In the remainder of this section, we shall describe a numerical method to calculate $\phi^{*}$ and the function $g(\cdot)$.

CASE II: $\lambda_{1}>\lambda_{0}$ and $0<\phi_{d}<\phi_{r}$. For every real number $\phi>\phi_{d}$, denote by $h_{\phi}:\left[\phi_{d}, \infty\right) \mapsto \mathbb{R}$ the unique solution in $\mathbf{C}\left(\left[\phi_{d}, \infty\right)\right) \cap \mathbf{C}^{1}\left(\left[\phi_{d}, \phi\right) \cup(\phi, \infty)\right)$ of

$$
\begin{gathered}
h_{\phi}^{\prime}(y)=-\lambda_{0} l(y) h_{\phi}(r y)-\operatorname{sgn}(\lambda+a y)|\lambda+a y|^{-b / a-1}(c \alpha y-\lambda), \quad y \in\left[\phi_{d}, \phi\right), \\
h_{\phi}(y)=0, \quad y \in[\phi,+\infty),
\end{gathered}
$$

where $l(y) \triangleq \operatorname{sgn}(\lambda+a y)|\lambda+a y|^{-b / a-1}|\lambda+a r y|^{b / a}$ is well-defined for every $y \in\left[\phi_{d}, \infty\right)$ because $a<0$ and $r>1$; see (13).

Proposition 3.3. Let $\phi^{*}$ and $g(\cdot)$ be as in Proposition 3.2. The function $h_{\phi^{*}}(\cdot)$ is the only one among all $h_{\phi}$, $\phi>\phi_{d}$ such that

$$
f_{-1}(y) \triangleq-|\lambda+a y|^{-b / a} \leq h_{\phi}(y) \leq 0, \quad \forall y \in\left[\phi_{d}, \infty\right)
$$

By defining it on $\left(0, \phi^{*}\right)$ as the solution of the differential equation (23), its extension onto $\mathbb{R}_{+}$(denoted also by $\left.h_{\phi^{*}}\right)$ remains between the same bounds of $(25)$ on $\mathbb{R}_{+}$. We have $g(y)=|\lambda+a y|^{b / a} h_{\phi^{*}}(y)$ for every $y \in \mathbb{R}_{+}$, and

$$
\phi_{r}<\phi^{*}<\bar{\phi} \triangleq \frac{r}{c \alpha} \cdot\left[\frac{b-a}{r^{-(b / a)+1}-1}+(b-a-c \alpha) \cdot \frac{\lambda}{b} \cdot \frac{r^{-b / a}-1}{r^{-(b / a)+1}-1}\right] .
$$

The function $I(\phi) \triangleq h_{\phi}\left(\phi_{d}\right), \phi \in\left[\phi_{r}, \infty\right)$ is continuous and strictly decreasing, and $I\left(\phi^{*}\right)=0$.

Thanks to Proposition 3.3, one can find $\phi^{*}$ (and $h_{\phi^{*}}(\cdot)$ on $\left[\phi_{d}, \infty\right)$ ) by the bisection search in the interval $\left(\phi_{r}, \bar{\phi}\right)$ : Initially, let $\left(\phi_{0}, \bar{\phi}_{0}\right)=\left(\phi_{r}, \bar{\phi}\right)$. For every $n \geq 0$, let $\phi_{n}$ be the mid-point of the interval $\left[\underline{\phi}_{n}, \bar{\phi}_{n}\right]$; if $I\left(\phi_{n}\right)<0$, then set $\left(\bar{\phi}_{n+1}, \bar{\phi}_{n+1}\right)=\left(\phi_{n}, \phi_{n}\right)$, otherwise $\left(\phi_{n+1}, \bar{\phi}_{n+1}\right)=\left(\phi_{n}, \bar{\phi}_{n}\right)$. Then $\left\{\phi^{*}\right\}=\bigcap_{n \geq 0}\left[\phi_{n}, \bar{\phi}_{n}\right]$. Unfortunately, the solution $h_{\phi}$ of (23), (24) is unavailable in closed form; however, it can be calculated on $\left[\phi_{r}, \phi\right]$ fairly accurately by the finite difference methods. After $\phi^{*}$ and $h_{\phi^{*}}$ on $\left[\phi_{d}, \infty\right)$ are found, $h_{\phi^{*}}$ on $\left[0, \phi_{d}\right)$ can be calculated similarly from (23) by the continuation process (see, e.g., Bellman and Cooke [4, p. 47]). See Figure 2(a) for an illustration.

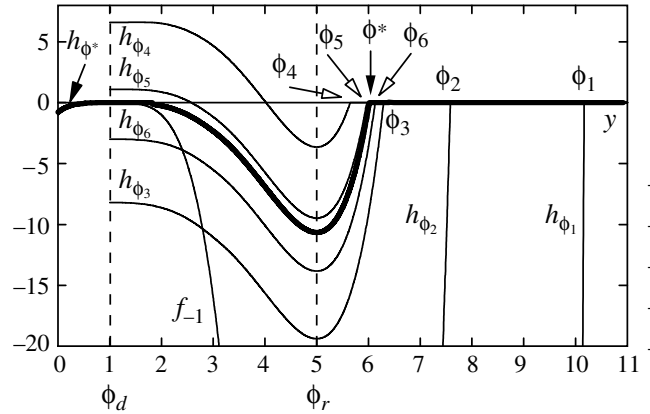

(a)

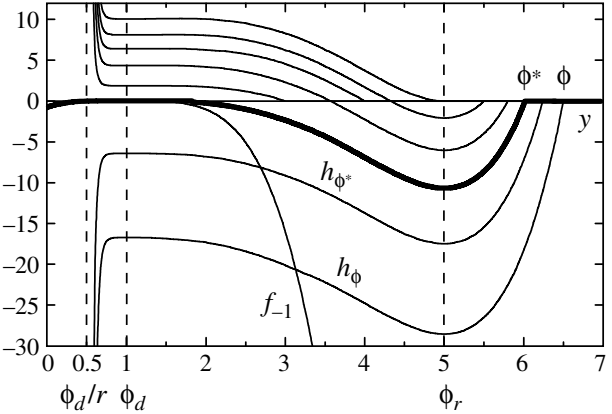

(b)

Figure 2. Case II. (a) bisection search for $\phi^{*}$, (b) comparison of $h_{\phi}(\cdot)$ for different $\phi$-values; see $(23,24)$. For both illustrations, the parameters are $\lambda=1, \lambda_{0}=3, \lambda_{1}=6, \alpha=1$, and $c=0.2$; we compute $r=2, \phi_{d}=1, \phi_{r}=5$; see (13). In (a), the search for $\phi^{*}$ starts in $\left[\phi_{r}, \bar{\phi}\right]=[5,15.33]$ with the tight upper bound $\bar{\phi}$ of Proposition 3.3, and continues along the intervals $\left[\phi_{r}, \phi_{1}\right] \supset\left[\phi_{r}, \phi_{2}\right] \supset\left[\phi_{r}, \phi_{3}\right] \supset$ $\left[\phi_{4}, \phi_{3}\right] \supset\left[\phi_{5}, \phi_{3}\right] \supset\left[\phi_{5}, \phi_{6}\right] \cdots$. The mid-points of the intervals are $\phi_{1}, \phi_{2}, \ldots$; the lefthand (righthand, resp.) half of each interval is eliminated if $I\left(\phi_{i}\right) \triangleq h_{\phi_{i}}\left(\phi_{d}\right)$ is positive (negative, resp.). The unique root of $I\left(\phi^{*}\right)=0$ is found at $\phi^{*}=6.016$ up to three decimal points after 17 iterations. The figure in (b) illustrates that the function $h_{\phi^{*}}(\cdot)$ is the separatrix for $(23,24)$ : among all $h_{\phi}, \phi \in\left[\phi_{d}, \infty\right)$, it is the only function bounded between $y$-axis and $y \mapsto f_{-1}(y)$ of Proposition 3.3. Moreover, $h_{\phi^{*}}\left(\phi_{d}\right)=0$, and $h_{\phi}\left(\phi_{d}\right)$ is positive (negative, resp.) for every $\phi<\phi^{*}\left(\phi>\phi^{*}\right.$, resp.); the functions $g_{\phi}(y) \triangleq|\lambda+a y|^{b / a} h_{\phi}(y)$ explode near $y=\phi_{d}$ for all $\phi$ but $\phi^{*}$. If $a+b<0$, equivalently $1<r \leq 2+(2 \lambda+\alpha) / \lambda_{0}$, then only $h_{\phi^{*}}$ has a stable extension onto $\mathbb{R}_{+}$; the functions $y \mapsto h_{\phi}(y)$ above it (below it, resp.) increase to $+\infty$ (decrease to $-\infty$, resp.) as $y$ decreases to $\phi_{d} / r$. (In both (a) and (b), the solutions $h_{\phi}(\cdot)$ of the differential equation $(23,24)$ of advanced type are computed by the finite difference method.) 
CASE III: $\lambda_{1}<\lambda_{0}$. For every real number $\beta$, let $\hbar_{\beta}: \mathbb{R}_{+} \mapsto \mathbb{R}$ be the unique continuously differentiable solution of

$$
\begin{gathered}
\hbar_{\beta}^{\prime}(y)=-(\lambda+a y)^{-b / a-1}\left[\lambda_{0}(\lambda+a r y)^{b / a} \hbar_{\beta}(r y)+c \alpha y-\lambda\right], \quad y>0, \\
\hbar_{\beta}(0)=\beta .
\end{gathered}
$$

The differential equations in (23) and (26) are essentially the same (in the latter case, $\lambda+a y$ is positive for every $y \in \mathbb{R}_{+}$because $a$ is positive). However, the solution $h_{\phi}(y)$ of (23) is unique if it is initially described for all $y \in[\phi, r \phi)$, whereas $\hbar_{\beta}(0)$ uniquely determines the solution $\hbar_{\beta}(\cdot)$ of (26). Strictly speaking, (23) of Case II is a differential equation of advanced type $\left(r=\lambda_{1} / \lambda_{0}>1\right)$, and (26) of Case III is a differential equation of retarded type $\left(r=\lambda_{1} / \lambda_{0}<1\right)$, see Bellman and Cooke [4, p. 48]. The integral equation obtained from (26) also resembles a renewal equation; this will be useful in the next section.

Proposition 3.4. Let $\phi^{*}$ and $g(\cdot)$ be as in Proposition 3.2. Then $g(y)=(\lambda+a y)^{b / a} \hbar_{\beta^{*}}(y)$ for every $y \in$ $\left[0, \phi^{*}\right)$, where $\beta^{*}$ is the unique number satisfying both $\hbar_{\beta^{*}}\left(\phi^{*}\right)=\hbar_{\beta^{*}}^{\prime}\left(\phi^{*}\right)=0$ and

$$
f_{-1}(y) \triangleq-(\lambda+a y)^{-b / a} \leq \hbar_{\beta^{*}}(y) \leq 0, \quad \forall y \in\left[0, \phi^{*}\right] .
$$

We have $\phi_{r}<\phi^{*}<b / c \alpha$ and $-\lambda^{-b / a}<\beta^{*}<0$. The function $J(\beta) \triangleq \max _{y \in[0, b / c \alpha]} \hbar_{\beta}(y), \beta \in\left[-\lambda^{-b / a}, 0\right]$ is continuous and strictly increasing, and $J\left(\beta^{*}\right)=0$.

One can find $\beta^{*}$ in Proposition 3.4 by bisection search in the interval $\left(\beta_{0}, \bar{\beta}_{0}\right)=\left(-\lambda^{-b / a}, 0\right)$ : For every $n \geq 0$, let $\beta_{n}$ be the mid-point of $\left(\underline{\beta}_{n}, \bar{\beta}_{n}\right)$. If $J\left(\beta_{n}\right)<0$, then let $\left(\underline{\beta}_{n+1}, \bar{\beta}_{n+1}\right)=\left(\beta_{n}, \bar{\beta}_{n}\right)$, otherwise $\left(\underline{\beta}_{n+1}, \bar{\beta}_{n+1}\right)=$ $\left(\underline{\beta}_{n}, \beta_{n}\right)$. Then $\left\{\beta^{*}\right\}=\bigcap_{n \geq 0}\left[\underline{\beta}_{n}, \bar{\beta}_{n}\right]$. See Figure 3(a). As the proof of Proposition 3.4 on page 229 reveals that the maximum $\phi^{*}$ at which $J\left(\beta^{*}\right)=\max _{x \in[0, b / c \alpha]} \hbar_{\beta^{*}}(x)$ is attained is unique in $[0, b / c \alpha]$.

REMARK 3.1. If the discount rate $\alpha$ decreases to zero in such a way that $C \triangleq c \alpha$ remains constant, then the Bayes error $R_{t}(\pi)$ of (5) converges for every $t \in \mathbb{R}_{+}$to $\hat{R}_{t}(\pi) \triangleq \mathbb{P}_{\pi}(\theta>t)+C \mathbb{E}_{\pi}\left[(t-\theta)^{+}\right]$, where the detection delay cost is proportional to the delay time. Peskir and Shiryaev [12] showed that the Poisson disorder problem $\hat{V}(\pi) \triangleq \inf _{\tau \in \mathscr{F}_{X}} \hat{R}_{\tau}(\pi)$ accepts a Bayes optimal stopping rule in the form of $\tau_{*} \triangleq \inf \left\{t \geq 0: \Pi_{t} \geq B_{*}\right\}$ for some suitable constant $B_{*} \in(0,1)$. Our results are in agreement with their findings. As $\alpha \searrow 0$, we have $\Phi_{t} \rightarrow \Psi_{t} \triangleq \Pi_{t} /\left(1-\Pi_{t}\right)$ for every $t \in \mathbb{R}_{+}$almost surely (see (10) and (11)); moreover, since $C \equiv c \alpha$ remains constant, we have $\phi_{r}=\lambda / C, \phi_{d}=-\lambda / a$ with $a=\lambda-\lambda_{1}+\lambda_{0}$ in (13). By Propositions 3.1 and 3.2, there is a suitable $\phi^{*} \geq \phi_{r}$ such that $\tau_{*} \triangleq \inf \left\{t \geq 0: \Psi_{t} \geq \phi^{*}\right\}$ is a minimum Bayes stopping rule. Equivalently, $\tau_{*}=\inf \left\{t \geq 0: \Pi_{t} \geq B_{*}\right\}$ with $B_{*} \triangleq \phi^{*} /\left(1+\phi^{*}\right)$, since $x \mapsto x /(1+x), x \in \mathbb{R}_{+}$is increasing. Propositions 3.1, 3.3, and 3.4 describe how to find $\phi^{*}$ (therefore, $B_{*}$ ) and the value function for different parameter ranges. For example, if $\lambda_{1} \geq \lambda_{0}$, and either $\phi_{d}<0$ or $0<\phi_{r} \leq \phi_{d}$ (equivalently, $\lambda>\lambda_{1}-\lambda_{0}$ or $C \geq \lambda_{1}-\lambda_{0}-\lambda$ ), then $\phi^{*}=\phi_{r}$ and $B_{*}=\lambda /(\lambda+C)$ by Proposition 3.1 (compare with Peskir and Shiryaev [12, Theorem 4.1]).

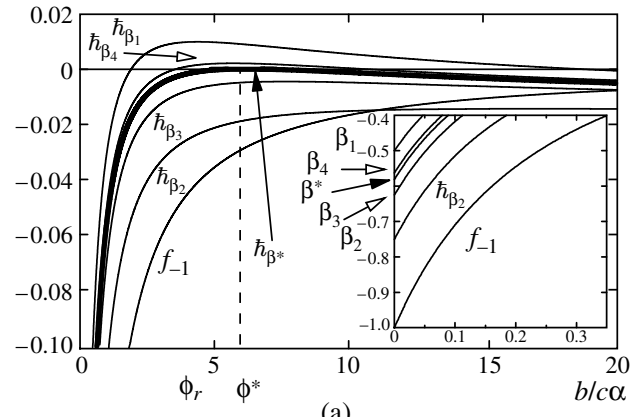

(a)

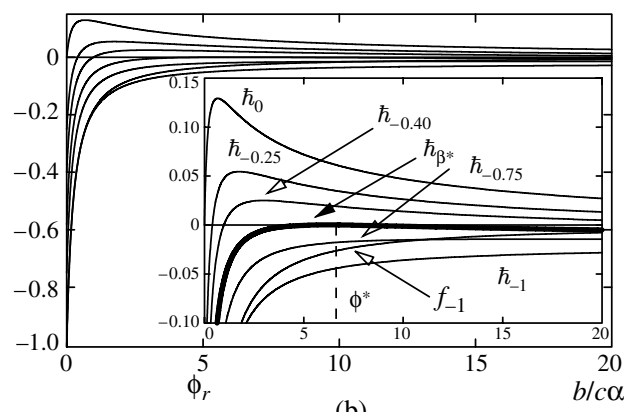

(b)

Figure 3. Case III. (a) bisection search for $\beta^{*}$, (b) comparison of $\hbar_{\beta}(\cdot)$ for different $\beta$-values, see $(26,27)$. For both illustrations, the parameters are $\lambda=1, \lambda_{0}=3, \lambda_{1}=1.5, \alpha=1$, and $c=0.2$; we compute $r=1 / 2, \phi_{d}<0, \phi_{r}=5$, see (13). By Proposition 3.4, $\phi_{r}<\phi^{*}<b / c \alpha=20$. The condition (28) on $\hbar_{\beta^{*}}$ implies that $-\lambda^{-b / a}=f_{-1}(0) \leq h_{\beta^{*}}(0)=\beta^{*} \leq 0$. Therefore, our search for $\beta^{*}$ starts in $\left[-\lambda^{-b / a}, 0\right]=[-1,0]$; it continues along the intervals $\left[-1, \beta_{1}\right] \supset\left[\beta_{2}, \beta_{1}\right] \supset\left[\beta_{3}, \beta_{1}\right] \supset\left[\beta_{3}, \beta_{4}\right] \cdots$ where $\beta_{1}, \beta_{2}, \ldots$ are the mid-points of the intervals; the lefthand (righthand, resp.) half of each interval is eliminated if $J\left(\beta_{i}\right) \triangleq \max _{y \in[0, b / c \alpha]} \hbar_{\beta_{i}}(y)$ is negative (positive, resp.). The unique zero of $J(\beta)=0$ in $[-1,0]$ is found after 10 iterations at $\beta^{*}=-0.581$ up to three decimal points; $J\left(\beta^{*}\right)$ is attained at $y=\phi^{*} \approx 6.196$. The figure in (b) displays the functions $\hbar_{\beta}$ for $\beta=-1,-0.75,-0.581\left(=\beta^{*}\right),-0.40,-0.25,0$. The function $\hbar_{\beta^{*}}$ is the smallest among all functions $\hbar_{\beta}, \beta \in\left[-\lambda^{-b / a}, 0\right]$ which intersect the $y$-axis. 
4. Proofs of Propositions 3.2, 3.3, and 3.4. In Case II, we prove Propositions 3.2 and 3.3 simultaneously by showing that there is a suitable $\phi^{*}>\phi_{d}$ such that the solution $h_{\phi^{*}}$ of $(23,24)$ with $\phi=\phi^{*}$ is bounded as in (25), and the function $g(y) \triangleq|\lambda+a y|^{b / a} h_{\phi^{*}}(y), y \in \mathbb{R}_{+}$has the desired properties enlisted in Proposition 3.2. In Case III, we prove Propositions 3.2 and 3.4 similarly. For the proofs of several lemmas below, it will be crucial to notice that the family of the auxiliary functions

$$
f_{m}(y) \triangleq m|\lambda+a y|^{-b / a}, \quad y \in \mathbb{R}_{+}, \quad m \in \mathbb{R} \text { solves } f^{\prime}(y)=-b l(y) f(r y), \quad y \in \mathbb{R}_{+} \backslash\left\{\phi_{d}\right\},
$$

which resembles Equations (23) and (26) that every $h_{\phi}$ and $\hbar_{\beta}$ satisfy, respectively. For every $m \in \mathbb{R}$, it is also easy to check that

$$
f_{m}(x)=f_{m}(R)-\int_{x}^{R} b|\lambda+a y|^{-b / a-1}|\lambda+a r y|^{b / a} f_{m}(r y) d y, \quad \phi_{d} \leq x \leq R<\infty .
$$

4.1. Case II: $\lambda_{1}>\lambda_{0}$ and $0<\phi_{d}<\phi_{r}$. For every $\phi>\phi_{d}$, let $h_{\phi}:\left[\phi_{d}, \infty\right) \mapsto \mathbb{R}$ be the unique solution of $(23,24)$ in $\mathbf{C}\left(\left[\phi_{d}, \infty\right)\right) \cap \mathbf{C}^{1}\left(\left[\phi_{d}, \phi\right) \cup(\phi, \infty)\right)$. The proof of the existence and the uniqueness of the function $h_{\phi^{*}}$, bounded as in (25), for some $\phi^{*}>\phi_{d}$ is broken down into several lemmas below. Lemmas 4.3 and 4.4 identify the crucial property of the family of functions $\left\{h_{\phi}\right\}$ : for any $\phi>\phi_{r}$, if $y \mapsto h_{\phi}(y)$ violates one of the bounds in (25), then $h_{\phi}\left(\phi_{d}\right) \neq 0$ (see also Figure 2(b)). However, the continuous mapping $\phi \mapsto I(\phi) \triangleq h_{\phi}\left(\phi_{d}\right)$ has unique root in $\left(\phi_{r}, \infty\right)$ (identified with $\left.\phi^{*}\right)$ by Lemmas 4.1, 4.2, 4.5, and 4.6. Therefore, $h_{\phi^{*}}$ becomes one (and the only) member of $\left\{h_{\phi}\right\}_{\phi>\phi_{r}}$ between the bounds in (25).

LEMma 4.1. If $\phi_{r} \leq A<B<\infty$, then $h_{A}(x)>h_{B}(x)$ for every $x \in\left[\phi_{d}, B\right)$.

Proof. It is sufficient to consider $\max \left\{\phi_{d}, B / r\right\} \leq A<B$; in general, $A \in\left[B / r^{n+1}, B / r^{n}\right.$ ) for some $n \in \mathbb{N}$, and $h_{A}>h_{B / r^{n}}>\cdots>h_{B / r}>h_{B}$ on $\left[\phi_{d}, B\right)$ follows. By (23) and (24), the difference $h_{A B} \triangleq h_{A}-h_{B}$ is positive on $\left[\max \left\{\phi_{d}, B / r\right\}, B\right]$. If $\phi_{d} \in[B / r, B)$, then the proof is complete. Otherwise, $h_{A B}$ satisfies

$$
\begin{gathered}
h_{A B}^{\prime}(y)=-\lambda_{0} l(y) h_{A B}(r y), \quad \phi_{d}<y<A, \\
h_{A B}(x)=h_{A B}(R)-\int_{x}^{R} \lambda_{0}|\lambda+a y|^{-b / a-1}|\lambda+\operatorname{ary}|^{b / a} h_{A B}(r y) d y, \quad \phi_{d} \leq x \leq R \leq A .
\end{gathered}
$$

There exists a positive real number $m_{1}$ such that $f_{m_{1}}(B / r)=h_{A B}(B / r)$. Since $f_{m_{1}}$ of (29) is increasing, and $h_{A B}$ is decreasing on $[B / r, B], f_{m_{1}}$ dominates $h_{A B}$ over $[B / r, B]$ (see Figure 4(a)). Therefore, a comparison of (30) and (32) with $R=B / r$ gives

$$
0 \leq f_{m_{1}}(x)<h_{A B}(x), \quad \forall x \in\left[B / r^{2}, B / r\right] .
$$

If $\phi_{d} \in\left[B / r^{2}, B / r\right]$, then the proof is complete. Otherwise, there exists a (positive) real number $m_{2}>m_{1}$ such that $f_{m_{2}}\left(B / r^{2}\right)=h_{A B}\left(B / r^{2}\right)>f_{m_{1}}\left(B / r^{2}\right)$. Since $f_{m_{2}}>f_{m_{1}}>h_{A B}$ on $[B / r, B]$, the comparison of the differential equations in (29) and (31) gives $f_{m_{2}}^{\prime}>h_{A B}^{\prime}>0$ on $\left[B / r^{2}, B / r\right]$. Because $f_{m_{2}}\left(B / r^{2}\right)=h_{A B}\left(B / r^{2}\right)$, this implies $f_{m_{2}}(x)>h_{A B}(x)$, for every $x \in\left[B / r^{2}, B / r\right]$ (see Figure 4(a)). Therefore, a comparison of (30) and (32) with $R=B / r^{2}$ gives

$$
0 \leq f_{m_{2}}(x)<h_{A B}(x), \quad \forall x \in\left[B / r^{3}, B / r^{2}\right]
$$

Because $\phi_{d} \in\left[B / r^{n}, B / r^{n-1}\right]$ for some $n$, from a finite induction the proof follows.

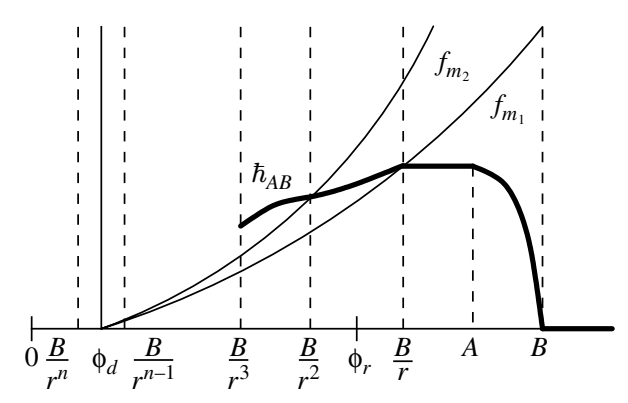

(a)

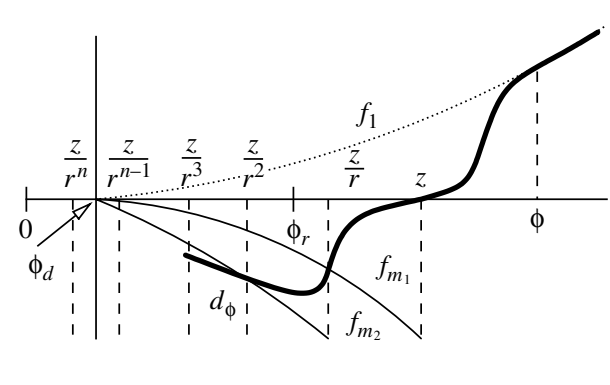

(b)

Figure 4. Illustrations for the proofs of (a) Lemma 4.1 and (b) Lemma 4.4. The location of $\phi_{r}$ is unimportant as long as $\phi_{d}<\phi_{r}<A<B$ in (a), and $\phi_{d}<\phi_{r}<\phi$ in (b). 
Lemma 4.2. If $\phi_{d} \leq A<B \leq \phi_{r}$, then $h_{A}(x)<h_{B}(x)$ for every $x \in\left[\phi_{d}, B\right)$. For every $\phi \in\left[\phi_{d}, \phi_{r}\right], h_{\phi}>0$ on $\left[\phi_{d}, \phi\right)$.

Proof. The proof of that $h_{B A} \triangleq h_{B}-h_{A}>0$ on $\left[\phi_{d}, B\right)$ is similar to that of Lemma 4.1. For the second part, observe that $\phi_{d} \in\left[\phi / r^{n+1}, \phi / r^{n}\right)$ for some $n \in \mathbb{N}$, and $h_{\phi / r^{n}} \geq 0$. Therefore, the first part implies $h_{\phi}>$ $h_{\phi / r}>\cdots>h_{\phi / r^{n}} \geq 0$ on $\left[\phi_{d}, \phi\right)$.

Lemma 4.3. Let $\phi \in\left(\phi_{r}, \infty\right)$. If $h_{\phi}(z)=0$ for some $z \in\left(\phi_{d}, \phi\right)$, then $h_{\phi}>0$ on $\left[\phi_{d}, z\right)$.

Proof. Let $\phi \in\left(\phi_{r}, \infty\right)$. Suppose that $h_{\phi}$ has some zeros in $\left(\phi_{d}, \phi\right)$, and let $z$ be the largest. By (23) and (24), $h_{A}<0$ on $\left[\max \left\{A / r, \phi_{r}\right\}, A\right)$ for every $A>\phi_{r}$. Since $\phi_{r} \in\left[\phi / r^{n+1}, \phi / r^{n}\right)$ for some $n \in \mathbb{N}$, Lemma 4.1 implies that $0>h_{\phi / r^{n}}>\cdots>h_{\phi / r}>h_{\phi}$ on $\left[\phi_{r}, \phi\right)$. Therefore, $z \in\left(\phi_{d}, \phi_{r}\right)$. Take any $A \in\left[\max \left\{\phi_{d}, z / r\right\}, z\right)$. With minor modifications to the proof of Lemma 4.2, it can be shown that $h_{\phi}>h_{A} \geq 0$ on $\left[\phi_{d}, z\right)$.

Lemma 4.4. Let $\phi \in\left(\phi_{r}, \infty\right)$. If $h_{\phi}(z)=f_{-1}(z)$ of (29) for some $z \in\left(\phi_{d}, \infty\right)$, then $h_{\phi}<f_{-1} \leq 0$ on $\left[\phi_{d}, z\right)$.

Proof. The function $d_{\phi}(x) \triangleq h_{\phi}(x)-f_{-1}(x), x \in\left[\phi_{d}, \infty\right)$ satisfies

$$
\begin{aligned}
& d_{\phi}^{\prime}(x)=-\lambda_{0} l(x) d_{\phi}(r x)-\operatorname{sgn}(\lambda+a x)|\lambda+a x|^{-b / a-1} c \alpha x, \quad x \in\left(\phi_{d}, \phi\right), \\
& d_{\phi}(x)=|\lambda+a x|^{-b / a}, \quad x \in[\phi, \infty) .
\end{aligned}
$$

Suppose that $d_{\phi}$ has some zeros in $\left(\phi_{d}, \infty\right)$, and let $z$ be the largest (note that $z \in\left(\phi_{d}, \phi\right)$ ). Since $d_{\phi}>0$ on $(z, \infty)$, (33) implies that $d_{\phi}^{\prime}>0$ and $d_{\phi}<0$ on $[z / r, z]$. If $\phi_{d} \in[z / r, z)$, then the proof is complete. Otherwise, (33) implies

$$
d_{\phi}(x)<d_{\phi}(R)+\int_{x}^{R} \lambda_{0} l(y) d_{\phi}(r y) d y, \quad \phi_{d} \leq x<R \leq z .
$$

There exists a negative real number $m_{1}$ such that $f_{m_{1}}(z / r)=d_{\phi}(z / r)$. Since $f_{m_{1}}$ is decreasing, and $d_{\phi}$ is increasing on $[z / r, z]$, we have $f_{m_{1}}<d_{\phi}$ on $[z / r, z]$. Therefore, (34) with $R=z / r$ implies (see Figure 4(b))

$$
d_{\phi}(x)<f_{m_{1}}(z / r)+\int_{x}^{z / r} \lambda_{0} l(z) f_{m_{1}}(r z) d z=f_{m_{1}}(x) \leq 0, \quad x \in\left[z / r^{2}, z / r\right] .
$$

If $\phi_{d} \in\left[z / r^{2}, z / r\right)$, then the proof is complete. Otherwise, there exists a negative real number $m_{2}<m_{1}$ such that $f_{m_{2}}\left(z / r^{2}\right)=d_{\phi}\left(z / r^{2}\right)<f_{m_{1}}\left(z / r^{2}\right)$. Since $f_{m_{2}}<f_{m_{1}}$, we have $f_{m_{2}}<d_{\phi}$ on $[z / r, z]$. Therefore, (33) implies

$$
d_{\phi}^{\prime}(x) \geq-\lambda_{0} l(x) d_{\phi}(r x) \geq-b l(x) f_{m_{2}}(r x)=f_{m_{2}}^{\prime}(x), \quad x \in\left[z / r^{2}, z / r\right] .
$$

Since $f_{m_{2}}\left(z / r^{2}\right)=d_{\phi}\left(z / r^{2}\right)$, this implies $f_{m_{2}}<d_{\phi}$ on $\left[z / r^{2}, z / r\right]$. Therefore, (34) (with $\left.R=z / r^{2}\right)$ implies $d_{\phi}<$ $f_{m_{2}} \leq 0$ on $\left[z / r^{3}, z / r^{2}\right]$. Since $\phi_{d} \in\left[z / r^{n}, z / r^{n-1}\right]$ for some $n \in \mathbb{N}$, a finite induction completes the proof.

LeMma 4.5. For every

$$
\phi \geq \bar{\phi} \triangleq \frac{r}{c \alpha} \cdot\left[\frac{b-a}{r^{-(b / a)+1}-1}+(b-a-c \alpha) \cdot \frac{\lambda}{b} \cdot \frac{r^{-b / a}-1}{r^{-(b / a)+1}-1}\right]>\phi_{r},
$$

we have $h_{\phi}<0$ on $\left[\phi_{d}, \phi\right)$.

PRoOF. If $h_{\phi}(\phi / r) \leq f_{-1}(\phi / r)<0$ for some $\phi \in\left(\phi_{r}, \infty\right)$, then $h_{\phi}<0$ on $\left[\phi_{d}, \phi\right)$ by Lemmas 4.3 and 4.4. Therefore, Lemma 4.1 implies that $0 \geq h_{\phi}>h_{\phi^{\prime}}$ on $\left[\phi_{d}, \phi^{\prime}\right)$ for every $\phi^{\prime}>\phi$. By $(23,24)$, we have

$$
h_{\phi}(\phi / r)=-\int_{\phi / r}^{\phi}|\lambda+a x|^{-b / a-1}(c \alpha x-\lambda) d x=-k(\phi)|\lambda+a \phi|^{-b / a}+k(\phi / r)|\lambda+a(\phi / r)|^{-b / a},
$$

where $k(\phi) \triangleq[b c \alpha \phi-\lambda(b-a-c \alpha)] /[b(b-a)]$. Therefore, $h_{\phi}(\phi / r) \leq f_{-1}(\phi / r)$ for some $\phi>\phi_{r}$ if and only if $[1+k(\phi / r)] / k(\phi) \leq|\lambda+a \phi|^{-b / a} /|\lambda+a(\phi / r)|^{-b / a}$. As $\phi$ tends to $\infty$, the function on the righthand side decreases to $r^{-b / a}$. Finally, the solution of $[1+k(\phi / r)] / k(\phi)=r^{-b / a}$ yields $\phi=\bar{\phi}$. One can also see from $0<\phi_{d}<\phi_{r}$ that $\bar{\phi}>\phi_{r}$.

Lemma 4.6. The function $I(\phi) \triangleq h_{\phi}\left(\phi_{d}\right), \phi \in\left[\phi_{r}, \infty\right)$ is strictly decreasing and continuous. 
Proof. For any $\phi_{r} \leq A<B<\infty$, the mapping $h_{A B} \triangleq h_{A}-h_{B}$ is positive on $\left[\phi_{d}, A\right)$ by Lemma 4.1. Therefore, $I(A)-I(B)=h_{A B}\left(\phi_{d}\right)>0$, i.e., $I(\cdot)$ is strictly decreasing. Because $h_{A B}=-h_{B} \geq 0$ on $[A, \infty), h_{A B}$ is nonnegative on $\left[\phi_{d}, \infty\right)$; therefore, $h_{A B}^{\prime}(y)=-\lambda_{0} l(y) h_{A B}(r y) \geq 0, y \in\left[\phi_{d}, A\right)$, thanks to (23). Hence, $h_{A B}$ is nondecreasing on $\left[\phi_{d}, A\right)$. If $\max \left\{\phi_{r}, B / r\right\}<A<B$, then

$$
0<I(A)-I(B)=h_{A B}\left(\phi_{d}\right) \leq h_{A B}(A)=\int_{A}^{B}|\lambda+a y|^{-b / a-1}(c \alpha y-\lambda) d y,
$$

where the last equality follows from (23) and (27). Because the integral above goes to zero as $B-A$ decreases to zero, the continuity of $I(\cdot)$ follows.

Proof of Proposition 3.2 in Case II and Proposition 3.3. By Lemma 4.6, the function $I(\cdot)$ introduced in Proposition 3.3 is continuous and strictly decreasing on $\left[\phi_{r}, \infty\right)$. Since $I(\phi)>0$ for every $\phi \in\left[\phi_{d}, \phi_{r}\right]$ by Lemma 4.2 , and $I(\bar{\phi})<0$ by Lemma 4.5 , the intermediate value theorem implies that there is unique $\phi^{*} \in\left(\phi_{r}, \bar{\phi}\right)$ such that $I\left(\phi^{*}\right)=0$. Lemmas 4.3 and 4.4 imply that $\phi^{*}$ and $h_{\phi^{*}}$ are the only solutions of (23), (24) that also satisfy (25). (If $\phi>\phi^{*}$, then $h_{\phi}\left(\phi_{d}\right)=I(\phi)<0=f_{-1}\left(\phi_{d}\right)$, i.e., $h_{\phi}(\cdot)$ violates the lower bound $f_{-1}(\cdot)$ in some neighborhood of $\phi_{d}$. If $\phi_{d}<\phi<\phi^{*}$, then $h_{\phi}\left(\phi_{d}\right)=I(\phi)>0$, i.e., $h_{\phi}(\cdot)$ violates the upper bound (namely, the nonpositivity) in some neighborhood of $\phi_{d}$.) We extend $h_{\phi^{*}}$ onto $\mathbb{R}_{+}$by defining it on $\left(0, \phi_{d}\right)$ as the solution of

$$
h_{\phi^{*}}^{\prime}(y)=-\lambda_{0} l(y) h_{\phi^{*}}(r y)-\operatorname{sgn}(\lambda+a y)|\lambda+a y|^{-b / a-1}(c \alpha y-\lambda), \quad y \in\left(0, \phi_{d}\right),
$$

where $l(y) \triangleq \operatorname{sgn}(\lambda+a y)|\lambda+a y|^{-b / a-1}|\lambda+\operatorname{ary}|^{b / a}$ as in (23). Because $h_{\phi^{*}} \leq 0$ on $\left[\phi_{d}, \infty\right)$ and $0<\phi_{d}<\phi_{r}$, (35) implies that $h_{\phi^{*}}^{\prime} \geq 0$ on $\left(0, \phi_{d}\right)$. Because $h_{\phi^{*}}\left(\phi_{d}\right)=0$, this yields that $h_{\phi^{*}} \leq 0$ on $\left(0, \phi_{d}\right)$. Because $f_{-1} \leq h_{\phi^{*}}$ on $\left[\phi_{d}, \infty\right)$ where $f_{-1}$ is given by (29), (35) also implies

$$
\begin{aligned}
h_{\phi^{*}}^{\prime}(y) & \leq-\lambda_{0} l(y) f_{-1}(r y)-\operatorname{sgn}(\lambda+a y)|\lambda+a y|^{-b / a-1}(c \alpha y-\lambda) \\
& =-b l(y) f_{-1}(r y)-\operatorname{sgn}(\lambda+a y)|\lambda+a y|^{-b / a-1} c \alpha y \leq-b l(y) f_{-1}(r y)=f_{-1}^{\prime}(y),
\end{aligned}
$$

for all $y \in\left(0, \phi_{d}\right)$. Because $f_{-1}\left(\phi_{d}\right)=h_{\phi^{*}}\left(\phi_{d}\right)=0$, this implies $f_{-1} \leq h_{\phi^{*}}$ on $\left(0, \phi_{d}\right)$. Thus, we showed

$$
f_{-1}(y) \triangleq-|\lambda+a y|^{-b / a} \leq h_{\phi^{*}}(y) \leq 0, \quad \forall y \in \mathbb{R}_{+} .
$$

The proof of Proposition 3.3, as well as that of Proposition 3.2 in Case II, is now complete because a direct computation using (23), (24) (with $\phi=\phi^{*}$ ) and (35) shows that the continuous function

$$
g(y) \triangleq \begin{cases}|\lambda+a y|^{b / a} h_{\phi^{*}}(y), & y \in \mathbb{R}_{+} \backslash\left\{\phi_{d}\right\}, \\ \lim _{x \rightarrow \phi_{d}}|\lambda+a x|^{b / a} h_{\phi^{*}}(x), & y=\phi_{d},\end{cases}
$$

which is evidently in $\mathbf{C}^{1}\left(\mathbb{R}_{+} \backslash\left\{\phi_{d}, \phi^{*}\right\}\right)$ and bounded in $[-1,0]$ by (36), satisfies (together with $\left.\phi^{*}\right)$ the conditions of the verification lemma on page 221 (an application of L'Hospital rule reveals that $g\left(\phi_{d}\right)$ is finite).

4.2. Case III: $\lambda_{1}<\lambda_{0}$. In this case, note that $a>0$ and $\phi_{d}<0$. Lemma 4.7 and its corollary establish the existence and the uniqueness of the solutions $\hbar_{\beta}$ of (26), (27). Lemmas 4.8, 4.9, and 4.10 are essential for the existence of unique $\hbar_{\beta^{*}}$ that lies between the bounds in (28). Lemma 4.11 hints that the search for the optimal stopping threshold $\phi^{*}$ in Propositions 3.2 and 3.4 can be confined into $\left(\phi_{r}, b / c \alpha\right]$.

Lemma 4.7. If $f, \eta$, and $\xi$ are real-valued functions on $\mathbb{R}_{+}$such that $|f(t)| \leq k, 0 \leq t \leq T$ and $\max _{0 \leq s \leq t}|\eta(s)| \leq \xi(t)$ with $\int_{0}^{T} \xi(s) d s<\infty$ for some positive constants $k$ and $T$, then

$$
u(t)=f(t)+\int_{0}^{t} \eta(s) u(r s) d s, \quad t \in[0, T]
$$

has unique integrable solution $u$. If $f$ is continuous (resp., continuously differentiable, and $\eta$ is continuous), then $u$ is continuous (resp., continuously differentiable). Moreover, if $\xi$ is bounded, so is $u$.

Proof. The proof is a slight modification of that of the existence and uniqueness of the renewal equation (Bellman and Cooke [4, p. 217]). 
COROLlary 4.1. Equations (26), (27) have unique bounded and continuously differentiable solution on $[0, T]$, for every $T>0$.

Proof. We can write (26), (27) as $\hbar_{\beta}(x)=f(x)+\int_{0}^{x} \eta(y) h(r y) d y$, with

$$
f(x) \triangleq \hbar_{\beta}(0)-\int_{0}^{x}(\lambda+a y)^{-b / a-1}(c \alpha x-\lambda) d y \quad \text { and } \quad \eta(x) \triangleq-\lambda_{0}(\lambda+a x)^{-b / a-1}(\lambda+a r x)^{b / a}
$$

Because $|\eta(y)|$ is decreasing, $\xi(x) \triangleq \max _{0 \leq y \leq x}|\eta(y)|=|\eta(0)|=1 / \lambda$ is bounded. Because $\eta$ is continuous (note that $a>0$ if $\lambda_{1}<\lambda_{0}$ ), and $f$ is continuously differentiable, the conclusion follows from Lemma 4.7.

LEMMA 4.8. If $\gamma>\beta$, then $\hbar_{\gamma}(x)>\hbar_{\beta}(x)$ for $x \in[0, T]$ for every $T>0$.

Proof. By means of the family of functions $f_{m}$ in (29), we shall prove that $h_{\gamma \beta} \triangleq \hbar_{\gamma}-\hbar_{\beta}$ is positive on $[0, T]$ for any fixed $T>0$. Using (26), (27), we find that

$$
\hbar_{\gamma \beta}^{\prime}(y)=-\lambda_{0} l(y) \hbar_{\gamma \beta}(r y), \quad y \in[0, T], \quad \text { and } \quad \hbar_{\gamma \beta}^{\prime}(0)=\gamma-\beta
$$

where $l(y) \triangleq(\lambda+a y)^{-b / a-1}(\lambda+r a y)^{b / a}$ as in (29). If $m_{0}$ is chosen such that $f_{m_{0}}(0)=\hbar_{\gamma \beta}(0)$, then (29) and (39) imply that $f_{m_{0}}^{\prime}(0)=-b l(0) f_{m_{0}}(0)=-b l(0) \hbar_{\gamma \beta}(0)<-\lambda_{0} l(0) \hbar_{\gamma \beta}(0)=\hbar_{\gamma \beta}^{\prime}(0)$. Hence $f_{m_{0}}<\hbar_{\gamma \beta}$ in some neighborhood of 0 . Therefore, there are some $m_{1}>m_{0}$ and $\varepsilon>0$ such that $f_{m_{1}}>\hbar_{\gamma \beta}>0$ on $[0, \varepsilon)$ and $f_{m_{1}}(\varepsilon)=$ $\hbar_{\gamma \beta}(\varepsilon)$. The proof is complete if $T \in(0, \varepsilon]$. Otherwise, note that $f_{m_{1}}^{\prime}(x)=-b l(x) f_{m_{1}}(r x) \leq-b l(x) \hbar_{\gamma \beta}(r x)<$ $-\lambda_{0} l(x) \hbar_{\gamma \beta}(r x)=\hbar_{\gamma \beta}^{\prime}(x)$ for every $x \in(\varepsilon, \varepsilon / r]$, and

$$
0<f_{m_{1}}(x)=f_{m_{1}}(\varepsilon)+\int_{\varepsilon}^{x} f_{m_{1}}^{\prime}(y) d y<\hbar_{\gamma \beta}(\varepsilon)+\int_{\varepsilon}^{x} \hbar_{\gamma \beta}^{\prime}(y) d y=\hbar_{\gamma \beta}(x), \quad x \in(\varepsilon, \varepsilon / r] .
$$

If $T \in(0, \varepsilon / r]$, then the proof is complete. Otherwise, we choose $m_{2}>m_{1}$ such that $f_{m_{2}}(\varepsilon / r)=\hbar_{\gamma \beta}(\varepsilon / r)>$ $f_{m_{1}}(\varepsilon / r)$, see Figure 5(a). Since $f_{m_{2}}>f_{m_{1}}$, we have $f_{m_{2}}>\hbar_{\gamma \beta}$ on $[0, \varepsilon]$. Therefore, $f_{m_{2}}^{\prime}(x)=-b l(x) f_{m_{2}}(r x) \leq$ $-b l(x) \hbar_{\gamma \beta}(r x)<-\lambda_{0} l(x) \hbar_{\gamma \beta}(r x)=\hbar_{\gamma \beta}^{\prime}(x)$ for every $x \in\left(\varepsilon / r, \varepsilon / r^{2}\right]$, and

$$
0<f_{m_{2}}(x)=f_{m_{2}}(\varepsilon / r)+\int_{\varepsilon / r}^{x} f_{m_{2}}^{\prime}(y) d y<\hbar_{\gamma \beta}(\varepsilon / r)+\int_{\varepsilon / r}^{x} \hbar_{\gamma \beta}^{\prime}(y) d y=\hbar_{\gamma \beta}(x), \quad x \in\left(\varepsilon / r, \varepsilon / r^{2}\right] .
$$

Because $T \in\left(\varepsilon / r^{n-1}, \varepsilon / r^{n}\right]$ for some $n \in \mathbb{N}$, the proof follows by finite induction.

LeMma 4.9. $\hbar_{\beta}(x)$ is a continuous function of $\beta \in \mathbb{R}$ uniformly in $x \in[0, T]$ for every $T>0$.

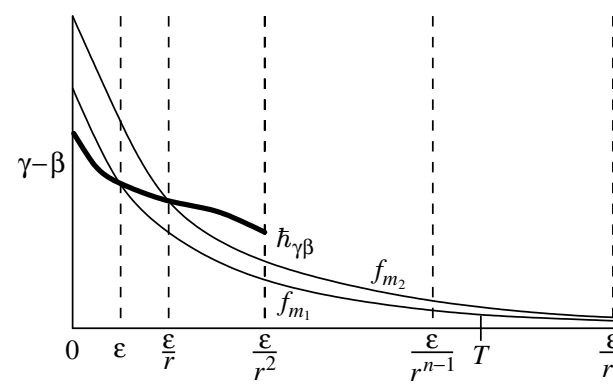

(a)

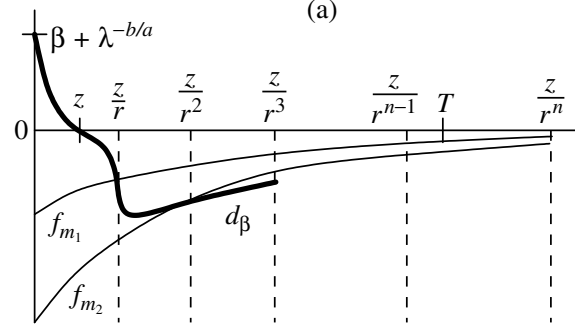

(b)

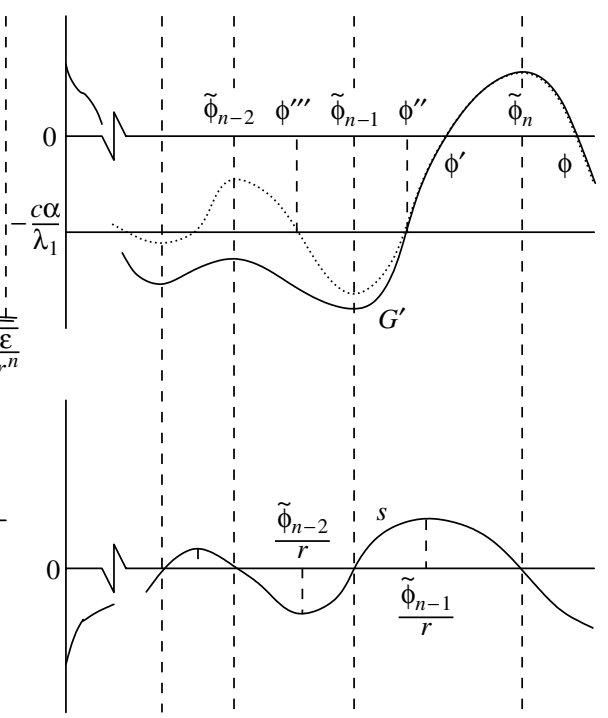

(c)

FIGURE 5. Illustrations for the proofs of (a) Lemma 4.8, (b) Lemma 4.10, and (c) Proposition 3.4. 
Proof. Let $\hbar_{\gamma \beta} \triangleq \hbar_{\gamma}-\hbar_{\beta}$. By (26) and (27), $\hbar_{\gamma}(x)=\gamma-\beta-\int_{0}^{x} \lambda_{0} l(y) \hbar_{\gamma \beta}(r y) d y$ for every $x \in[0, T]$. Because $l(x)=(\lambda+a x)^{-b / a-1}(\lambda+a r x)^{b / a}$ is decreasing and $l(0)=1 / \lambda$, we have

$$
\left|\hbar_{\gamma \beta}(x)\right|-\frac{\lambda_{0}}{r \lambda} \int_{0}^{x}\left|\hbar_{\gamma \beta}(z)\right| d z \leq|\gamma-\beta|, \quad x \in[0, T] .
$$

After multiplying by $\exp \left[-\lambda_{0} x /(r \lambda)\right]$ and taking the integrals of both sides, we obtain $\int_{0}^{x}\left|\hbar_{\gamma \beta}(z)\right| d z \leq|\gamma-\beta|$. $\exp \left[-\lambda_{0} x /(r \lambda)\right] \int_{0}^{x} \exp \left[\lambda_{0} z /(r \lambda)\right] d z$. Therefore, (40) implies

$$
\begin{aligned}
\left|\hbar_{\gamma \beta}(x)\right| & \leq|\beta-\gamma|\left(1+\left[\lambda_{0} /(r \lambda)\right] \exp \left[\lambda_{0} /(r \lambda)\right] \int_{0}^{x} \exp \left[\lambda_{0} z /(r \lambda)\right] d z\right) \\
& =|\gamma-\beta| \exp \left[\lambda_{0} x /(r \lambda)\right] \leq|\gamma-\beta| \exp \left[\lambda_{0} T /(r \lambda)\right], \quad \forall x \in[0, T] .
\end{aligned}
$$

Lemma 4.10. Let $\beta \in\left[-\lambda^{-b / a}\right.$, 0]. If $\hbar_{\beta}(z)=f_{-1}(z)$ for some $z \in \mathbb{R}_{+}$, then $\hbar_{\beta}(x)<f_{-1}(x)<0$ for every $x \in(z, T]$ and $T>z$, where $f_{-1}$ is as in (29) and $f_{-1}(0)=-\lambda^{-b / a}$.

Proof. First, let $\beta \in\left(-\lambda^{-b / a}, 0\right]$. Suppose that $d_{\beta}(x) \triangleq \hbar_{\beta}(x)-f_{-1}(x), x \in \mathbb{R}_{+}$has some zeros, and let $z$ be the smallest $(z>0)$. We shall prove that $d_{\beta}<0$ on $(z, T]$ for every $T>0$. By (26), (27), and (29),

$$
d_{\beta}^{\prime}(x)=-\lambda_{0} l(x) d_{\beta}(r x)-(\lambda+a x)^{-b / a-1} c \alpha x, \quad x \in \mathbb{R}_{+} \quad \text { and } \quad d_{\beta}(0)=\beta+\lambda^{-b / a} .
$$

Because $d_{\beta}>0$ on $[0, z),(41)$ implies that $d_{\beta}$ is decreasing on $[0, z / r]$. Hence, $d_{\beta}<0$ on $(z, z / r]$. If $T \in(z, z / r]$, then the proof is complete. Otherwise, there exists some $m_{1}<0$ such that $f_{m_{1}}(z / r)=d_{\beta}(z / r)$. Since $f_{m_{1}}$ is increasing, and $d_{\beta}$ is decreasing on $[0, z / r]$, we have $d_{\beta} \geq f_{m_{1}}$ on $[0, z / r]$. Therefore, $f_{m_{1}}^{\prime}(x)=-b l(x) f_{m_{1}}(r x) \geq$ $-b l(x) d_{\beta}(r x)>-\lambda_{0} l(x) d_{\beta}(r x)-(\lambda+a x)^{-b / a-1} c \alpha x=d_{\beta}^{\prime}(x)$ for every $x \in\left[z / r, z / r^{2}\right]$. Thus,

$$
0>f_{m_{1}}(x)=f_{m_{1}}(z / r)+\int_{z / r}^{x} f_{m_{1}}^{\prime}(y) d y>d_{\beta}(z / r)+\int_{z / r}^{x} d_{\beta}^{\prime}(y) d t=d_{\beta}(x), \quad x \in\left(z / r, z / r^{2}\right] .
$$

If $T \in\left(z / r, z / r^{2}\right]$, then the proof is complete. Otherwise, there exists $m_{2}<m_{1}$ such that $f_{m_{2}}\left(z / r^{2}\right)=d_{\beta}\left(z / r^{2}\right)<$ $f_{m_{1}}\left(z / r^{2}\right)$, see Figure 5(b). Because $f_{m_{2}}<f_{m_{1}}$, we have $f_{m_{2}}<d_{\beta}$, on $[z, z / r]$. By (29) and (41), $f_{m_{2}}^{\prime}(x)=$ $-b l(x) f_{m_{2}}(r x) \geq-b l(x) d_{\beta}(r x)>-\lambda_{0} l(x) d_{\beta}(r x)-(\lambda+a x)^{-b / a-1} c \alpha x=d_{\beta}^{\prime}(x)$ for every $x \in\left[z / r, z / r^{2}\right]$. Because $f_{m_{2}}\left(z / r^{2}\right)=d_{\beta}\left(z / r^{2}\right)$, this implies that $f_{m_{2}}<d_{\beta}$ on $\left[z / r, z / r^{2}\right)$. By using (29) and (41) once again, $f_{m_{2}}^{\prime}(x)=-b l(x) f_{m_{2}}(r x) \geq-b l(x) d_{\beta}(r x)>-\lambda_{0} l(x) d_{\beta}(r x)-(\lambda+a x)^{-b / a-1} c \alpha x=d_{\beta}^{\prime}(x)$ for every $x \in\left[z / r^{2}, z / r^{3}\right]$. Because $f_{m_{2}}\left(z / r^{2}\right)=d_{\beta}\left(z / r^{2}\right)$, this implies that $0>f_{m_{2}}>d_{\beta}$ on $\left(z / r^{2}, z / r^{3}\right]$. Because $T \in$ $\left(z / r^{n-1}, z / r^{n}\right]$ for some $n \in \mathbb{N}$, a finite induction argument concludes the proof. If $\beta=-\lambda^{-b / a}$, then $d_{\beta}(0)=$ $d_{\beta}^{\prime}(0+)=0$, and $d_{\beta}$ is decreasing on $[0, z / r]$ for some small $z>0$; the rest of the proof follows as above.

Lemma 4.11. The function $J(\beta) \triangleq \max _{x \in[0, b / c \alpha]} \hbar_{\beta}(x), \beta \in\left[-\lambda^{-b / a}, 0\right]$ is continuous and strictly increasing. There exists unique $\beta^{*} \in\left(-\lambda^{-b / a}, 0\right)$ such that $J\left(\beta^{*}\right)=0$. The maximum $J\left(\beta^{*}\right)$ is attained in $\left(\phi_{r}, b / c \alpha\right]$; at every $\phi \in\left(\phi_{r}, b / c \alpha\right]$ where it is attained, we have $\hbar_{\beta^{*}}(\phi)=\hbar_{\beta^{*}}^{\prime}(\phi)=0$.

Proof. Lemmas 4.8 and 4.9 imply that $J(\cdot)$ is strictly increasing and continuous on $\left[-\lambda^{-b / a}, 0\right]$. On the other hand, $J(0)>0$ since $\hbar_{0}(0)=0<\hbar_{0}^{\prime}(0+)$, and $J\left(-\lambda^{-b / a}\right) \leq \max _{x \in[0, b / c \alpha]} f_{-1}(x)=f_{-1}(b / c \alpha)<0$ by Lemma 4.10. By the intermediate value theorem, there exists unique $\beta^{*} \in\left(-\lambda^{-b / a}, 0\right)$ such that $0=J\left(\beta^{*}\right)=$ $\max _{x \in[0, b / c \alpha]} \hbar_{\beta^{*}}(x)$. Because $h_{\beta^{*}}(\cdot)$ is continuous by Lemma 4.9 and $h_{\beta^{*}}(0)=\beta^{*}<0$, the maximum $J\left(\beta^{*}\right)$ is attained in $(0, b / c \alpha]$. If it is attained at some $\phi \in(0, b / c \alpha)$, then $\hbar_{\beta^{*}}(\phi)=\hbar_{\beta^{*}}^{\prime}(\phi)=0$ is obvious. Suppose that $\hbar_{\beta^{*}}(b / c \alpha)=0$. Then $\hbar_{\beta^{*}}>f_{-1}$ on $[0, b / c \alpha]$ by Lemma 4.10 (otherwise, there exists some $z \in[0, b / c \alpha$ ) such that $\hbar_{\beta^{*}}(z)=f_{-1}(z)$, and $\hbar_{\beta^{*}}<f_{-1}<0$ on $\left.[z, b / c \alpha]\right)$. By (26) and (29),

$$
\hbar_{\beta^{*}}^{\prime}(x)=(\lambda+a x)^{-b / a-1}\left[\lambda_{0} \frac{\hbar_{\beta^{*}}(r x)}{f_{-1}(r x)}-(c \alpha x-\lambda)\right] \leq(\lambda+a x)^{-b / a-1}(b-c \alpha x) \leq 0, \quad \forall x \in[b / c \alpha, u),
$$

where $u \triangleq \inf \left\{x \in \mathbb{R}_{+}: \hbar_{\beta^{*}}(x) \leq f_{-1}(x)\right\}>b / c \alpha$, because both $\hbar_{\beta^{*}}$ and $f_{-1}$ are continuous. However, this implies that $\max _{x \in[0, u)} \hbar_{\beta^{*}}(x)=J\left(\beta^{*}\right)=\hbar_{\beta^{*}}(b / c \alpha)=0$ and $\hbar_{\beta^{*}}^{\prime}(b / c \alpha)=0$. Finally, let $\phi_{0} \in(0, b / c \alpha]$ be the smallest number where $J\left(\beta^{*}\right)$ is attained. Then $\hbar_{\beta}(y)<0$ for every $y<\phi_{0}$. If $\phi_{0} \leq \phi_{r}$, then (26) implies $\hbar_{\beta^{*}}^{\prime}\left(\phi_{0}\right)=-\left(\lambda+a \phi_{0}\right)^{-b / a-1}\left[\lambda_{0}\left(\lambda+a \phi_{0}\right)^{b / a} \hbar_{\beta^{*}}\left(r \phi_{0}\right)+c \alpha \phi_{0}-\lambda\right]>0$, which contradicts with $\hbar_{\beta^{*}}^{\prime}\left(\phi_{0}\right)=0$. Thus, $\phi_{0}>\phi_{r}$. 
Proof of Proposition 3.2 in Case III and Proposition 3.4. By Lemma 4.11, the function $J(\cdot)$ has the properties enlisted in Proposition 3.4. As in the same lemma, let $\beta^{*} \in\left(-\lambda^{-b / a}, 0\right)$ and $\phi^{*} \in\left(\phi_{r}, b / c \alpha\right]$ be such that $0=\hbar_{\beta^{*}}\left(\phi^{*}\right)=J\left(\beta^{*}\right) \triangleq \max _{x \in[0, b / c \alpha]} \hbar_{\beta^{*}}(x)$; by the choice of $\phi^{*}$, we have $\hbar_{\beta^{*}}^{\prime}\left(\phi^{*}\right)=0$ and

$$
f_{-1}(x) \triangleq-(\lambda+a y)^{-b / a}<\hbar_{\beta^{*}}(x) \leq 0, \quad \forall x \in\left[0, \phi^{*}\right]
$$

(If $f_{-1}(z)=\hbar_{\beta^{*}}(z)$ for some $z<\phi^{*}$, then $0>f_{-1}>\hbar_{\beta^{*}}$ on $\left[z, \phi^{*}\right]$ by Lemma 4.10, which contradicts with $\hbar_{\beta^{*}}\left(\phi^{*}\right)=0$.) We define

$$
g(y) \triangleq \begin{cases}(\lambda+a y)^{b / a} \hbar_{\beta^{*}}(y), & y \in\left[0, \phi^{*}\right), \\ 0, & y \in\left[\phi^{*}, \infty\right) .\end{cases}
$$

The function $g(\cdot)$ is continuous and continuously differentiable on $\mathbb{R}_{+}$(because $\hbar_{\beta^{*}}\left(\phi^{*}\right)=\hbar_{\beta^{*}}^{\prime}\left(\phi^{*}\right)=0, g(\cdot)$ is differentiable at $\phi^{*}$ ), and is bounded in $[-1,0]$ by $(42)$. The proofs of both propositions will be complete if we show that $g(\cdot)$ and $\phi^{*}$ satisfy the conditions of the verification lemma on page 221. Using (26) with $\phi=\phi^{*}$, direct computation shows that $g(\cdot)$ solves $\left(20^{\prime}, 21^{\prime}\right)$ and satisfies the inequality (22) for every $y \in$ $\left[0, \phi^{*}\right] \cup\left[\phi^{*} / r, \infty\right)$ (recall that $\phi^{*}>\phi_{r}$ ). It remains only to show that $g(\cdot)$ satisfies (22) for $y \in\left(\phi^{*}, \phi^{*} / r\right)$. Equivalently, because

$$
\begin{aligned}
0 & \leq(\lambda+a y) g^{\prime}(y)-b g(y)+\lambda_{0} g(r y)+c \alpha y-\lambda=\lambda_{0} g(r y)+c \alpha y-\lambda \\
& =\lambda_{0}(\lambda+a r y)^{b / a} \hbar_{\beta^{*}}(r y)+c \alpha y-\lambda \Longleftrightarrow \hbar_{\beta^{*}}^{\prime}(y) \leq 0, \quad \forall y \in\left(\phi^{*}, \phi^{*} / r\right)
\end{aligned}
$$

by the definition of $g(\cdot)$ and (26), it is sufficient to prove that $\hbar_{\beta^{*}}(\cdot)$ is decreasing on $\left[\phi^{*}, \phi^{*} / r\right]$. We define

$$
\begin{gathered}
G(y) \triangleq(\lambda+a y)^{b / a} \hbar_{\beta^{*}}(y), \\
k(y) \triangleq(\lambda+a y)^{-b / a+1} G^{\prime}(y) \quad \text { and } \quad s(y) \triangleq(\lambda+a y)^{-b / a+2} G^{\prime \prime}(y), \quad \forall y \in \mathbb{R}_{+} .
\end{gathered}
$$

Because $\hbar_{\beta^{*}}\left(\phi^{*}\right)=\hbar_{\beta^{*}}^{\prime}\left(\phi^{*}\right)=0$, and $\phi^{*}$ is a local maximum of $\hbar_{\beta^{*}}$, it is also a local maximum of $G(\cdot)$, and

$$
G\left(\phi^{*}\right)=G^{\prime}\left(\phi^{*}\right)=0 \quad \text { and } \quad G^{\prime \prime}\left(\phi^{*}\right)<0 .
$$

Note that $g(\cdot)$ and $G(\cdot)$ coincide on $\left[0, \phi^{*}\right)$. Unlike $g(\cdot), G(\cdot)$ satisfies everywhere on $y \in \mathbb{R}_{+}$

$$
\begin{gathered}
(\lambda+a y) G^{\prime}(y)-b G(y)+\lambda_{0} G(r y)+c \alpha y-\lambda=0, \\
(\lambda+a y) G^{\prime \prime}(y)-(b-a) G^{\prime}(y)+\lambda_{1} G^{\prime}(r y)+c \alpha=0, \\
(\lambda+a y) G^{\prime \prime \prime}(y)-(b-2 a) G^{\prime \prime}(y)+\lambda_{1} r G^{\prime \prime}(r y)=0,
\end{gathered}
$$

where the last two equations follow from the first by differentiation. After they are multiplied by $(\lambda+a y)^{-(b-a) / a}$ and $(\lambda+a y)^{-(b-2 a) / a}$, respectively, and their terms are rearranged by using $k(\cdot)$ and $s(\cdot)$ in $(45)$, we obtain $(51)$ and (52) below. In (50), we rewrite the dynamics (26) of $\hbar_{\beta^{*}}$ in terms of $G(\cdot)$ :

$$
\begin{gathered}
\hbar_{\beta^{*}}^{\prime}(y)=-(\lambda+a y)^{-b / a-1}\left[\lambda_{0} G(r y)+c \alpha y-\lambda\right], \\
k^{\prime}(y)=-(\lambda+a y)^{-b / a}\left[\lambda_{1} G^{\prime}(r y)+c \alpha\right], \\
s^{\prime}(y)=-\lambda_{1} r(\lambda+a y)^{-b / a+1} G^{\prime \prime}(r y)=-\lambda_{1} r(\lambda+a y)^{-b / a+1}(\lambda+a r y)^{b / a-2} s(r y) .
\end{gathered}
$$

Because $\hbar_{\beta^{*}}^{\prime}\left(\phi^{*}\right)=0,(50)$ implies $\lambda_{0} G\left(r \phi^{*}\right)+c \alpha \phi^{*}-\lambda=0$. Thus, $\hbar_{\beta}(\cdot)$ is decreasing on $\left[\phi^{*}, \phi^{*} / r\right]$ if the mapping $y \mapsto \lambda_{0} G(r y)+c \alpha y-\lambda$ is increasing on $\left[\phi^{*}, \phi^{*} / r\right]$, equivalently, its derivative $\lambda_{1} G^{\prime}(r y)+c \alpha$ is positive at every $y \in\left[\phi^{*}, \phi^{*} / r\right]$. Therefore, the verification will be finalized under (46) when we show that

$$
\text { if } G^{\prime}(\phi)=0 \text { and } G^{\prime \prime}(\phi)<0 \text {, then } G^{\prime}(y)>-c \alpha / \lambda_{1} \text { for every } y \in[r \phi, \phi] \text {. }
$$

By the second equality in (52), the function $s(\cdot)$ reverts itself to the mean-level $y=0$. By $(42),-1 \leq G(\cdot) \leq 0$, and (45) imply that $s(0)<0$. Let $\tilde{\phi} \triangleq 0$, and $0<\tilde{\phi}_{1}<\widetilde{\phi}_{2}<\cdots$ be the intersection points of $s(\cdot)$ with $y$-axis (if there are finitely many of them, then we set the rest to $+\infty$ ). Then (52) implies that $s(\cdot)$ is increasing (decreasing, resp.) on $\left(\widetilde{\phi}_{n} / r, \widetilde{\phi}_{n+1} / r\right)$ for every even (odd, resp.) $n \in \mathbb{N}$, and

$$
\tilde{\phi}_{0} \triangleq 0<\tilde{\phi}_{1}<\frac{\tilde{\phi}_{1}}{r}<\tilde{\phi}_{2}<\frac{\tilde{\phi}_{2}}{r}<\tilde{\phi}_{3}<\frac{\tilde{\phi}_{3}}{r}<\cdots<\tilde{\phi}_{n-1}<\frac{\tilde{\phi}_{n-1}}{r}<\tilde{\phi}_{n}<\cdots .
$$


Because $s(\cdot)$ and $G^{\prime \prime}(\cdot)$ have the same signs, $G^{\prime \prime}$ is negative (positive, resp.) on $\left(\widetilde{\phi}_{n}, \widetilde{\phi}_{n+1}\right)$ for every even (odd, resp.) $n \in \mathbb{N}$. Therefore, $G^{\prime}(\cdot)$ is decreasing (increasing, resp.) on $\left(\widetilde{\phi}_{n}, \widetilde{\phi}_{n+1}\right)$ for every even (odd, resp.) $n \in \mathbb{N}$; see Figure 5(c).

Let $\phi \in \mathbb{R}_{+}$be such that $G^{\prime}(\phi)=0$ and $G^{\prime \prime}(\phi)<0$. Then $\phi \in\left(\tilde{\phi}_{n}, \tilde{\phi}_{n+1}\right)$ for some even $n \in \mathbb{N}$, and $G^{\prime}(y) \geq$ $G^{\prime}(\phi)=0$ for every $y \in\left[\tilde{\phi}_{n}, \phi\right]$. If $n=0$, then $G^{\prime}>0$ on $[0, \phi)$ and (53) follows. Suppose $n \geq 2$. If $G^{\prime}(\cdot) \geq$ $-c \alpha / \lambda_{1}$ on $\left[\widetilde{\phi}_{n-1}, \widetilde{\phi}_{n}\right]$, then (53) holds because $[r \phi, \phi] \subseteq\left[r \widetilde{\phi}_{n}, \phi\right] \subseteq\left[\widetilde{\phi}_{n-1}, \phi\right]$ by (54), and $G^{\prime} \geq 0>-c \alpha / \lambda_{1}$ on $\left[\widetilde{\phi}_{n}, \phi\right]$. For the rest, we suppose that $G^{\prime}(y)=-c \alpha / \lambda_{1}$ for some $y \in\left[\tilde{\phi}_{n-1}, \widetilde{\phi}_{n}\right]$. Because $G^{\prime}(\cdot)$ is increasing on $\left[\widetilde{\phi}_{n-1}, \widetilde{\phi}_{n}\right]$, it intersects with $y=-c \alpha / \lambda_{1}$ and $y=0$ exactly once, say at $\phi^{\prime \prime}$ and $\phi^{\prime}$, respectively. Then, $\tilde{\phi}_{n-1} \leq \phi^{\prime \prime}<\phi^{\prime}<\tilde{\phi}_{n}<\phi$. We prove (53) by showing that $\phi^{\prime \prime}<r \phi$ (indeed, this implies $[r \phi, \phi] \subset\left[\phi^{\prime \prime}, \phi\right]$; because $G^{\prime}(\cdot)>-c \alpha / \lambda_{1}$ on $\left[\phi^{\prime \prime}, \phi\right],(53)$ follows).

Observe that $k(\cdot)$ in (45) and $G^{\prime}(\cdot)$ have exactly the same zeros and signs. Thus, $k\left(\phi^{\prime}\right)=k(\phi)=0$ and $k(\cdot)>0$ on $\left(\phi^{\prime}, \phi\right)$. Therefore, $k(\cdot)$ has at least one local maximum in $\left(\phi^{\prime}, \phi\right)$. Hence, there exists at least one $y \in\left(\phi^{\prime}, \phi\right)$ such that (i) $k^{\prime}(y)=0$, (ii) $k^{\prime}(\cdot)>0$ on $(y-\varepsilon, y)$, and (iii) $k^{\prime}(\cdot)<0$ on $(y, y+\varepsilon)$ for some $\varepsilon>0$. Then (51) and (54) imply that

$$
\begin{gathered}
\text { there exists at least one } z \in\left[r \phi^{\prime}, r \phi\right] \subseteq\left[\tilde{\phi}_{n-2}, \tilde{\phi}_{n}\right] \\
\text { such that } G^{\prime}(z)=-c \alpha / \lambda_{1} \text {, and for some } \varepsilon>0, \\
G^{\prime}(y)<-c \alpha / \lambda_{1} \text { for } y \in(z-\varepsilon, z) \text { and } G^{\prime}(y)>-c \alpha / \lambda_{1} \text { for } y \in(z, z+\varepsilon) .
\end{gathered}
$$

In the interval $\left[\widetilde{\phi}_{n-1}, \widetilde{\phi}_{n}\right], \phi^{\prime \prime}$ is the only candidate for $z$ in $(55)$. On the other hand, even if $G^{\prime}(\cdot)$ intersects with $y=-c \alpha / \lambda_{1}$ on $\left[\widetilde{\phi}_{n-2}, \tilde{\phi}_{n-1}\right]$, the intersection point cannot become $z$ in (55) since $G^{\prime}(\cdot)$ is decreasing on $\left[\tilde{\phi}_{n-2}, \tilde{\phi}_{n-1}\right]$; see the dotted curve in Figure 5(c). Hence, $\phi^{\prime \prime}$ must be $z$ in (55). Therefore, $\phi^{\prime \prime} \leq r \phi$ and (53) is proved.

For the proof of the uniqueness of $\phi^{*}$, let us pick $\phi^{*}$ as the smallest of all numbers $\phi$ such that $\hbar_{\beta^{*}}(\phi)=$ $\hbar_{\beta^{*}}^{\prime}(\phi)=0$, and $g(\cdot)$ be as in (43). By (46) and (53), we have $\hbar_{\beta^{*}}^{\prime}(\cdot)<0$ on $\left(\phi^{*}, \phi^{*} / r\right)$, and (44) implies that (22) holds with strict inequality on $\left(\phi^{*}, \phi^{*} / r\right)$. An application of the chain-rule (see Lemma A.1) shows that $\left[\phi^{*}, \phi^{*} / r\right]$ must be in the optimal stopping region. Suppose that there exists another $\hat{\phi} \in \mathbb{R}_{+}$such that it is optimal to stop in $[\hat{\phi}, \infty)$. Then, we must have $\hat{\phi} \leq \phi^{*}$. Because $g(y)<0$ on $y \in\left[0, \phi^{*}\right)$, it is optimal not to stop in $\left[0, \phi^{*}\right)$; therefore $\hat{\phi} \geq \phi^{*}$. Thus, $\hat{\phi}=\phi^{*}$.

Appendix A.1. The Bayesian analysis. Let $\mathbb{Q}_{u}$ be the probability measure induced on $\left(\Omega, \mathscr{F}_{\infty}^{X}\right)$ by the finite-dimensional distribution of $X$ of (4) given that $\theta=u \in[0, \infty]$. Then

$$
\left.\frac{d \mathbb{Q}_{u}}{d \mathbb{Q}_{\infty}}\right|_{\mathscr{F}_{t}^{X}}=1_{\{u>t\}}+1_{\{u \leq t\}} \frac{L_{t}}{L_{u}}, \quad \text { and } \quad L_{t} \triangleq\left(\frac{\lambda_{1}}{\lambda_{0}}\right)^{X_{t}} e^{-\left(\lambda_{1}-\lambda_{0}\right) t}, \quad u, t \geq 0 .
$$

It is easily checked that $\mathbb{P}_{\pi}(F)=\pi \mathbb{Q}_{0}(F)+(1-\pi) \int_{0}^{\infty} \mathbb{Q}_{s}(F) \lambda e^{-\lambda s} d s$ for every $F \in \mathscr{F}_{\infty}^{X}$. Moreover, the useful identity

$$
\mathbb{P}_{\pi}(F \cap\{\theta>t\})=(1-\pi) \mathbb{Q}_{t}(F) e^{-\lambda t}, \quad \forall F \in \mathscr{F}_{t}^{X}, \quad \forall t \geq 0
$$

follows from the equality $\mathbb{P}_{\pi}(F \cap\{\theta>t\})=\int_{t}^{\infty} \mathbb{Q}_{s}(F) \mathbb{P}_{\pi}\{\theta \in d s\}$ and that the measure $\mathbb{Q}_{s}$ coincides with $\mathbb{Q}_{\infty}$ on $\mathscr{F}_{t}^{X}$ for every $s \geq t$. Using the generalized Bayes' theorem (see, e.g., Shiryaev [16, p. 230-231], Liptser and Shiryaev [9, p. 303-306]), we obtain

$$
\mathbb{P}_{\pi}\left\{\theta \leq s \mid \mathscr{F}_{t}^{X}\right\}=\frac{\pi L_{t}+(1-\pi)\left[\int_{0}^{s \wedge t}\left(L_{t} / L_{u}\right) \lambda e^{-\lambda u} d u+e^{-\lambda t}-e^{-\lambda(s \vee t)}\right]}{\pi L_{t}+(1-\pi)\left[\int_{0}^{t}\left(L_{t} / L_{u}\right) \lambda e^{-\lambda u} d u+e^{-\lambda t}\right]}, \quad \forall s, t \geq 0,
$$

and

$$
\begin{aligned}
& \mathbb{P}_{\pi}\left\{\theta=0 \mid \mathscr{F}_{t}^{X}\right\}=\frac{\pi L_{t}}{\pi L_{t}+(1-\pi)\left[\int_{0}^{t}\left(L_{t} / L_{u}\right) \lambda e^{-\lambda u} d u+e^{-\lambda t}\right]}, \quad t \geq 0, \\
& \mathbb{P}_{\pi}\left\{\theta \in d s \mid \mathscr{F}_{t}^{X}\right\}=\frac{(1-\pi)\left(L_{t} / L_{s}\right) \lambda e^{-\lambda s} d s}{\pi L_{t}+(1-\pi)\left[\int_{0}^{t}\left(L_{t} / L_{u}\right) \lambda e^{-\lambda u} d u+e^{-\lambda t}\right]}, \quad 0<s \leq t .
\end{aligned}
$$


By substituting $t$ for $s$ in (58), we obtain

$$
\begin{gathered}
\Pi_{t} \triangleq \mathbb{P}_{\pi}\left\{\theta \leq t \mid \mathscr{F}_{t}^{X}\right\}=\frac{\pi L_{t}+(1-\pi) \int_{0}^{t}\left(L_{t} / L_{u}\right) \lambda e^{-\lambda u} d u}{\pi L_{t}+(1-\pi)\left[\int_{0}^{t}\left(L_{t} / L_{u}\right) \lambda e^{-\lambda u} d u+e^{-\lambda t}\right]}, \\
\Psi_{t} \triangleq \frac{\Pi_{t}}{1-\Pi_{t}}=e^{\lambda t} L_{t}\left[\frac{\pi}{1-\pi}+\int_{0}^{t} \frac{1}{L_{u}} \lambda e^{-\lambda u} d u\right], \quad t \geq 0
\end{gathered}
$$

Using (59) and (60), we also calculate

$$
\mathbb{E}_{\pi}\left[1_{\{\theta \leq t\}} e^{\alpha(t-\theta)^{+}} \mid \mathscr{F}_{t}^{X}\right]=\frac{e^{\alpha t}\left[\pi L_{t}+(1-\pi) \int_{0}^{t}\left(L_{t} / L_{u}\right) \lambda e^{-(\alpha+\lambda) u} d u\right]}{\pi L_{t}+(1-\pi)\left[\int_{0}^{t}\left(L_{t} / L_{u}\right) \lambda e^{-\lambda u} d u+e^{-\lambda t}\right]},
$$

and

$$
\Phi_{t} \triangleq \frac{\mathbb{E}_{\pi}\left[1_{\{\theta \leq t\}} e^{\alpha(t-\theta)^{+}} \mid \mathscr{F}_{t}^{X}\right]}{1-\Pi_{t}}=e^{(\alpha+\lambda) t} L_{t}\left[\frac{\pi}{1-\pi}+\int_{0}^{t} \frac{1}{L_{u}} \lambda e^{-(\alpha+\lambda) u} d u\right], \quad t \geq 0 .
$$

Once noticed that $L_{t}$ in (56) obeys $d L_{t} / L_{t-}=-\left(\lambda_{1}-\lambda_{0}\right) d t+\left(\lambda_{1} / \lambda_{0}-1\right) d X_{t}, t \geq 0$ and $L_{0}=1$, the chain rule gives (10) and (11) as the dynamics of $\Psi_{t}$ in (62) and $\Phi_{t}$ in (63), respectively. Another application of the chain rule to $\Pi_{t}=\Psi_{t} /\left(1+\Psi_{t}\right)$ with (10) gives the dynamics of $\Pi$ as in (9).

Proof of (8). Let $\tau$ be an $\mathscr{F}^{X}$-stopping time. Since

$$
e^{\alpha(\tau-\theta)^{+}}-1=\int_{0}^{(\tau-\theta)^{+}} \alpha e^{\alpha u} d u=1_{\{\tau>\theta\}} \int_{\theta}^{\tau} \alpha e^{\alpha(s-\theta)} d s=\int_{0}^{\infty} 1_{\{\tau>s\}} 1_{\{\theta \leq s\}} \alpha e^{\alpha(s-\theta)^{+}} d s,
$$

the expectation of both sides gives

$$
\mathbb{E}_{\pi}\left[e^{\alpha(\tau-\theta)^{+}}-1\right]=\int_{0}^{\infty} \mathbb{E}_{\pi}\left(1_{\{\tau>s\}} \alpha \mathbb{E}_{\pi}\left[1_{\{\theta \leq s\}} e^{\alpha(s-\theta)^{+}} \mid \mathscr{F}_{s}^{X}\right]\right) d s=\mathbb{E}_{\pi} \int_{0}^{\tau}\left(1-\Pi_{s}\right) \alpha \Phi_{s} d s,
$$

because of Fubini's theorem, that $\{\tau>s\} \in \mathscr{F}_{s}^{X}$ for every $s \geq 0$ and (7). But

$$
\begin{aligned}
\mathbb{P}_{\pi}\{\theta>\tau\} & =\int_{0}^{\infty} \mathbb{Q}_{s}\{\tau<s\} \mathbb{P}_{\pi}\{\theta \in d s\}=\int_{0}^{\infty}\left[1-\mathbb{Q}_{s}\{\tau \geq s\}\right](1-\pi) \lambda e^{-\lambda s} d s \\
& =1-\pi-\int_{0}^{\infty} \lambda(1-\pi) \mathbb{Q}_{s}\{\tau \geq s\} e^{-\lambda s} d s=1-\pi-\int_{0}^{\infty} \lambda \mathbb{P}_{\pi}\{\tau \geq s, \theta>s\} d s \\
& =1-\pi-\int_{0}^{\infty} \lambda \mathbb{E}_{\pi}\left[1_{\{\tau \geq s\}}\left(1-\Pi_{s}\right)\right] d s=1-\pi-\mathbb{E}_{\pi} \int_{0}^{\tau} \lambda\left(1-\Pi_{s}\right) d s
\end{aligned}
$$

where the forth equality follows from (57) since $\{\tau \geq s\} \in \mathscr{F}_{s}^{X}$ for every $s \geq 0$. Finally, the sum of (64) and the preceding equation gives (8).

Presented here for completeness, the formulas (58)-(64) were derived for the first time by Shiryaev [14, 15, Chapter 4] and Beibel [3].

Appendix A.2. The chain-rule and the verification lemma. Needed at the end of this section on page 221 for the proof of the verification lemma, the lemmas in the remainder are concerned with the processes $\Pi$ and $\widetilde{\Phi}$ of $(15)$ on the probability space $\left(\Omega, \mathscr{F}, \mathbb{P}_{\pi, \phi}\right)$ for every $(\pi, \phi) \in[0,1] \times \mathbb{R}_{+}$, see page 221 . They remain true when $(\Pi, \tilde{\Phi})$ and $\mathbb{P}_{\pi, \phi}$ in their statements and in their proofs are replaced with $(\Pi, \Phi)$ and $\mathbb{P}_{\pi}$, respectively, because (i) the probability measures $\mathbb{P}_{\pi}$ and $\mathbb{P}_{\pi, \pi /(1-\pi)}$ are the same on $(\Omega, \mathscr{F})$, and (ii) the triplet $(X, \Pi, \widetilde{\Phi})$ under $\mathbb{P}_{\pi, \pi /(1-\pi)}$ has the same finite-dimensional distribution as that of the triplet $(\Omega, \Pi, \Phi)$ under $\mathbb{P}_{\pi}($ compare (15) and (11)). Finally, observe that the innovations process

$$
\bar{X}_{t} \triangleq X_{t}-\int_{0}^{t}\left[\lambda_{0}\left(1-\Pi_{s}\right)+\lambda_{1} \Pi_{s}\right] d s, \quad t \geq 0
$$

is an $\mathscr{F}^{X}$-martingale under both $\mathbb{P}_{\pi}$ and $\mathbb{P}_{\pi, \phi}$ for every $\pi \in[0,1]$ and $\phi \in \mathbb{R}_{+}$.

Lemma A.1. Let $G:[0,1] \times \mathbb{R}_{+} \mapsto \mathbb{R}$ be a continuous and piecewise continuously differentiable function in each coordinate. Then

$$
G\left(\Pi_{t}, \tilde{\Phi}_{t}\right)=G\left(\Pi_{0}, \tilde{\Phi}_{0}\right)+\int_{0}^{t} \mathscr{A} G\left(\Pi_{s}, \tilde{\Phi}_{s}\right) d s+M_{t}, \quad t \geq 0
$$


where $M_{t} \triangleq \int_{(0, t]} \Delta G\left(\Pi_{s-}, \tilde{\Phi}_{s-}\right) d \bar{X}_{s}, t \geq 0$ is an $\mathscr{F}^{X}$-local martingale; $\Delta G(\pi, \phi) \triangleq G\left(\lambda_{1} \pi /\left[\lambda_{0}(1-\pi)+\right.\right.$ $\left.\left.\lambda_{1} \pi\right],\left[\lambda_{1} / \lambda_{0}\right] \phi\right)-G(\pi, \phi)$ for every $(\pi, \phi) \in[0,1] \times \mathbb{R}_{+}$, and

$$
\begin{aligned}
\mathscr{A} G(\pi, \phi) \triangleq & \partial_{\pi} G(\pi, \phi)\left[\lambda-\left(\lambda_{1}-\lambda_{0}\right) \pi\right](1-\pi)+\partial_{\phi} G(\pi, \phi)\left[\lambda+\left(\lambda+\alpha-\lambda_{1}+\lambda_{0}\right) \phi\right] \\
& +\left[\lambda_{0}(1-\pi)+\lambda_{1} \pi\right] \Delta G(\pi, \phi)
\end{aligned}
$$

at every $(\pi, \phi) \in(0,1) \times \mathbb{R}_{+}$where the partial derivatives $\partial_{\pi} G$ and $\partial_{\phi} G$ exist. If $G$ is bounded, and $\tau$ is an $\mathscr{F}^{X}$-stopping time with finite $\mathbb{P}_{\pi, \phi^{-}}$expectation, then

$$
\mathbb{E}_{\pi, \phi} G\left(\Pi_{0}, \tilde{\Phi}_{0}\right)=\mathbb{E}_{\pi, \phi} G\left(\Pi_{\tau}, \tilde{\Phi}_{\tau}\right)-\mathbb{E}_{\pi, \phi} \int_{0}^{\tau} \mathscr{A} G\left(\Pi_{s}, \tilde{\Phi}_{s}\right) d s .
$$

Proof. Denote by $\Pi^{c}$ and $\widetilde{\Phi}^{c}$ the continuous parts of the processes $\Pi$ and $\widetilde{\Phi}$. Let

$$
\Delta \Pi_{t}=\frac{\left(\lambda_{1}-\lambda_{0}\right) \Pi_{t-}\left(1-\Pi_{t-}\right)}{\lambda_{0}\left(1-\Pi_{t-}\right)+\lambda_{1} \Pi_{t-}} \quad \text { and } \quad \Delta \tilde{\Phi}_{t}=\left(\lambda_{1} / \lambda_{0}-1\right) \tilde{\Phi}_{t-}, \quad t>0 .
$$

The processes $\Pi$ and $\tilde{\Phi}$ jump simultaneously with the jump sizes $\Delta \Pi_{t}$ and $\Delta \tilde{\Phi}_{t}$ at every time $t$ when $X$ jumps. Standard application of the chain-rule with the dynamics of $\Pi$ and $\widetilde{\Phi}$ in (9) and (15), respectively, gives $G\left(\Pi_{t}, \widetilde{\Phi}_{t}\right)=G\left(\Pi_{0}, \widetilde{\Phi}_{0}\right)+\int_{(0, t]} \mathscr{A} G\left(\Pi_{s-}, \widetilde{\Phi}_{s-}\right) d s+M_{t}$ where $\mathscr{A} G$ and $M_{t}$ are as above. This proves (66). Suppose next that $G$ is bounded, and $\tau$ is an $\mathscr{F}^{X}$-stopping time with finite expectation under $\mathbb{P}_{\pi, \phi}$. Because

$$
\mathbb{E}_{\pi, \phi} \int_{0}^{\tau}\left|\Delta G\left(\Pi_{s}, \tilde{\Phi}_{s}\right)\right|\left[\lambda_{1} \Pi_{s}+\lambda_{0}\left(1-\Pi_{s}\right)\right] d s \leq \sup _{p, y} 2|G(p, y)|\left(\lambda_{0} \vee \lambda_{1}\right) \mathbb{E}_{\pi, \phi} \tau<\infty,
$$

the stopped process $\left\{M_{t \wedge \tau}: t \geq 0\right\}$ is a closable $\mathscr{F}^{X}$-martingale under $\mathbb{P}_{\pi, \phi}$. Therefore, $\mathbb{E}_{\pi, \phi} M_{\tau}=0$. If we replace $\tau$ with $t$ and take expectations of both sides in (66), then (68) follows.

Lemma A.2. Let $\tau_{\phi} \triangleq \inf \left\{t \geq 0: \widetilde{\Phi}_{t} \geq \phi\right\}$ be the first exit time of the process $\tilde{\Phi}$ out of the interval $[0, \phi]$, $\phi \in \mathbb{R}_{+}$. Then $\mathbb{E}_{\pi, y} \tau_{\phi}$ is finite for every $(\pi, y) \in[0,1] \times \mathbb{R}_{+}$.

Proof. If $y \geq \phi$, then $\mathbb{E}_{\pi, y} \tau_{\phi}=0$. Suppose that $0 \leq y<\phi$. By (15) and (65), we obtain for every $t \in \mathbb{R}_{+}$

$$
\begin{aligned}
\tilde{\Phi}_{t \wedge \tau_{\phi}}-\tilde{\Phi}_{0} & =\int_{0}^{t \wedge \tau_{\phi}}\left[\lambda+\left(\lambda+\alpha-\lambda_{1}+\lambda_{0}\right) \tilde{\Phi}_{s}\right] d s+\int_{0}^{t \wedge \tau_{\phi}}\left(\lambda_{1} / \lambda_{0}-1\right) \tilde{\Phi}_{s-} d X_{s} \\
& =\int_{0}^{t \wedge \tau_{\phi}}\left\{\lambda+\left(\lambda+\alpha-\lambda_{1}+\lambda_{0}\right) \tilde{\Phi}_{s}+\left(\frac{\lambda_{1}}{\lambda_{0}}-1\right)\left[\lambda_{1} \Pi_{s}+\lambda_{0}\left(1-\Pi_{s}\right)\right] \tilde{\Phi}_{s}\right\} d s+M_{t \wedge \tau_{\phi}},
\end{aligned}
$$

where $M_{t \wedge \tau_{\phi}} \triangleq \int_{0}^{t \wedge \tau_{\phi}}\left(\lambda_{1} / \lambda_{0}-1\right) \tilde{\Phi}_{s-} d \bar{X}_{s}$ is a stopped $\mathscr{F}^{X}$-martingale under $\mathbb{P}_{\pi, y}$ because

$$
\mathbb{E}_{\pi, y} \int_{0}^{t \wedge \tau_{\phi}}\left|\left(\frac{\lambda_{1}}{\lambda_{0}}-1\right) \tilde{\Phi}_{s-}\right|\left[\lambda_{1} \Pi_{s}+\lambda_{0}\left(1-\Pi_{s}\right)\right] d s \leq \frac{\left|\lambda_{1}-\lambda_{0}\right| \phi}{\lambda_{0}}\left(\lambda_{1} \vee \lambda_{0}\right) \mathbb{E}_{\pi, y}\left(t \wedge \tau_{\phi}\right)<\infty, \quad \forall t \in \mathbb{R}_{+} .
$$

Therefore, $\mathbb{E}_{\pi, y} M_{t \wedge \tau_{\phi}}=0$ for every $t \in \mathbb{R}_{+}$. After the last integrand in (69) is simplified, and the expectations of both sides are taken, we obtain

$$
\mathbb{E}_{\pi, y} \tilde{\Phi}_{t \wedge \tau_{\phi}}-y=\mathbb{E}_{\pi, y} \int_{0}^{t \wedge \tau_{\phi}}\left[\lambda+\left(\lambda+\alpha+\frac{\left(\lambda_{1}-\lambda_{0}\right)^{2}}{\lambda_{0}} \Pi_{s}\right) \tilde{\Phi}_{s}\right] d s \geq \lambda \mathbb{E}_{\pi, y}\left(t \wedge \tau_{\phi}\right) .
$$

By (15), we have $\mathbb{P}_{\phi, y}\left\{\widetilde{\Phi}_{t \wedge \tau_{\phi}} \leq \phi \cdot \max \left(\lambda_{1} / \lambda_{0}, 1\right)\right\}=1$; therefore, $\mathbb{E}_{\pi, y}\left(t \wedge \tau_{\phi}\right) \leq\left(\mathbb{E}_{\pi, y} \widetilde{\Phi}_{t \wedge \tau_{\phi}}-y\right) / \lambda \leq[\phi$. $\left.\max \left(\lambda_{1} / \lambda_{0}, 1\right)-y\right] / \lambda$ for every $t \in \mathbb{R}_{+}$. When the limit of both sides is taken as $t$ tends to infinity, the conclusion follows from the monotone convergence theorem.

Proof of Verification Lemma. Suppose that $g: \mathbb{R}_{+} \mapsto(-\infty, 0]$ is bounded, continuous and piecewise continuously differentiable. Let $G(\pi, y) \triangleq(1-\pi) g(y)$ for every $(\pi, y) \in[0,1] \times \mathbb{R}_{+}$. For every $\pi \in[0,1]$ and $y \in \mathbb{R}_{+}$where $g^{\prime}(y)$ exists, we have $A(1-\pi) g(y)=(1-\pi)\left[(\lambda+a y) g^{\prime}(y)-b g(y)+\lambda_{0} g(r y)\right]$. For every $\mathscr{F}^{X}$-stopping time $\tau$ with finite $\mathbb{P}_{\pi, y}$-expectation (see pages 221 and 231), the last part of Lemma A.1 implies

$$
\begin{aligned}
(1-\pi) g(y) & =\mathbb{E}_{\pi, y} G\left(\Pi_{0}, \Phi_{0}\right) \\
& =\mathbb{E}_{\pi, y} G\left(\Pi_{\tau}, \Phi_{\tau}\right)-\mathbb{E}_{\pi, y} \int_{0}^{\tau}\left(1-\Pi_{s}\right)\left[\left(\lambda+a \Phi_{s}\right) g^{\prime}\left(\Phi_{s}\right)-b g\left(\Phi_{s}\right)+\lambda_{0} g\left(r \Phi_{s}\right)\right] d s \\
& \leq \mathbb{E}_{\pi, y} \int_{0}^{\tau}\left(1-\Pi_{s}\right)\left(c \alpha \tilde{\Phi}_{s}-\lambda\right) d s, \quad(\pi, y) \in[0,1] \times \mathbb{R}_{+},
\end{aligned}
$$


where the inequality follows from (22) and that $G$ is nonpositive. When we take the infimum of both sides in (70) over all $\mathscr{F}^{X}$-stopping times with finite $\mathbb{P}_{\pi, y}$-expectations, we obtain $(1-\pi) g(y) \leq \tilde{V}(\pi, y)$ for every $(\pi, y) \in[0,1] \times \mathbb{R}_{+}$.

Suppose that the same function $g$ above is in $\mathbf{C}\left(\mathbb{R}_{+}\right) \cap \mathbf{C}^{1}\left(\mathbb{R}_{+} \backslash\left\{\phi_{d}, \phi\right\}\right)$ for some real number $\phi>\phi_{r}$ and solves $\left(20^{\prime}, 21^{\prime}\right)$. By Lemma A.2, the $\mathscr{F}^{X}$-stopping time $\tau_{\phi} \triangleq \inf \left\{t \geq 0\right.$ : $\left.\tilde{\Phi}_{t} \geq \phi\right\}$ has finite $\mathbb{P}_{\pi, y}$-expectation. When $\tau$ is replaced with $\tau_{\phi},(70)$ is still true; but, now with an equality instead of the inequality (thanks to $\left(20^{\prime}\right)$ and $\left.\left(21^{\prime}\right)\right)$. Together with the inequality obtained in the first part, we have

$$
\tilde{V}(\pi, y)=(1-\pi) g(y)=\mathbb{E}_{\pi, y} \int_{0}^{\tau_{\phi}}\left(1-\Pi_{s}\right)\left(c \alpha \widetilde{\Phi}_{s}-\lambda\right) d s, \quad(\pi, y) \in[0,1] \times \mathbb{R}_{+},
$$

and $\tau_{\phi}$ is optimal for (16).

Acknowledgments. Erhan Bayraktar is supported by the U.S. Office of Naval Research under Grant N0001403-1-0102 and the U.S. Army Pantheon Project. Savas Dayanik is supported by the National Science Foundation under Grant NSF-DMI-04-23327. We thank the associate editor and the anonymous referee for their careful reading and their suggestions, which made our earlier presentation better.

\section{References}

[1] Bayraktar, Erhan, Savas Dayanik, Ioannis Karatzas. 2005. The standard Poisson disorder problem revisited. Stochastic Process. Appl. 115(9) 1437-1450.

[2] Beibel, M. 1996. A note on Ritov's Bayes approach to the minimax property of the CUSUM procedure. Ann. Statist. 24(4) 1804-1812.

[3] Beibel, M. 2000. A note on sequential detection with exponential penalty for the delay. Ann. Statist. 28(6) 1696-1701.

[4] Bellman, Richard, Kenneth L. Cooke. 1963. Differential-Difference Equations. Academic Press, New York.

[5] Bensoussan, Alain. 1992. Stochastic Control of Partially Observable Systems. Cambridge University Press, Cambridge, UK.

[6] Davis, M. H. A. 1976. A note on the Poisson disorder problem. Banach Center Publ. 1 65-72.

[7] Galchuk, L. I., B. L. Rozovskii. 1971. The disorder problem for a Poisson process. Theory Probab. Appl. 16 $729-734$.

[8] Karatzas, Ioannis. 2003. A note on Bayesian detection of change-points with an expected miss criterion. Statist. Decisions 21(1) 3-13.

[9] Liptser, Robert S., Albert N. Shiryaev. 2001. Statistics of Random Processes, I, expanded ed., Applications of Mathematics, Vol. 5. Springer-Verlag, Berlin, New York.

[10] Moustakides, G. V. 2004. Optimality of the CUSUM procedure in continuous time. Ann. Statist. 32(1).

[11] Peskir, G., A. N. Shiryaev. 2000. Sequential testing problems for Poisson processes. Ann. Statist. 28(3) 837-859.

[12] Peskir, G., A. N. Shiryaev. 2002. Solving the Poisson disorder problem. Advances in Finance and Stochastics. Springer, Berlin, $295-312$.

[13] Poor, H. Vincent. 1998. Quickest detection with exponential penalty for delay. Ann. Statist. 26(6) 2179-2205.

[14] Shiryaev, A. N. 1973. Statistical Sequential Analysis. American Mathematical Society, Providence, RI.

[15] Shiryaev, A. N. 1978. Optimal Stopping Rules. Springer-Verlag, New York.

[16] Shiryaev, A. N. 1996. Probability. Graduate Texts in Mathematics, Vol. 95. Springer-Verlag, New York.

[17] Shiryaev, A. N. 2002. Quickest detection problems in the technical analysis of the financial data. Mathematical finance-Bachelier Congress, 2000 (Paris). Springer Finance, Springer, Berlin, Germany, 487-521.

[18] Whittle, Peter. 1990. Risk-Sensitive Optimal Control. Wiley-Interscience Series in Systems and Optimization. John Wiley \& Sons Ltd., Chichester, UK.

[19] Whittle, Peter. 1996. Optimal Control. Wiley-Interscience Series in Systems and Optimization. John Wiley \& Sons Ltd., Chichester, UK. 